\title{
Pd-catalyzed transfer of difluorocarbene
}

\author{
Xiao-Yun Deng, Jin-Hong Lin and Ji-Chang Xiao* \\ Key Laboratory of Organofluorine Chemistry, Shanghai Institute of Organic Chemistry, University of Chinese \\ Academy of Sciences, Chinese Academy of Sciences, 345 Lingling Road, Shanghai 200032, China.
}

Fax: (+86) 21-6416-6128; Tel: (+86)21-5492-5340; E-mail: jchxiao@sioc.ac.cn

\section{Contents}

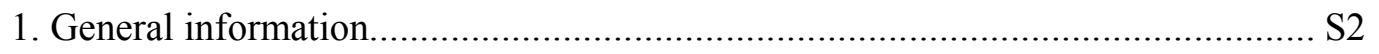

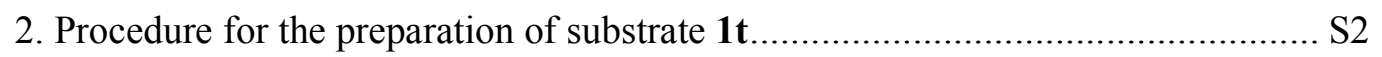

3. Screening reaction conditions for $\mathrm{CF}_{2}$-transfer reaction ............................... $\mathrm{S} 3$

4. General procedure for the Pd-catalyzed difluorocarbene transfer reaction........... S4

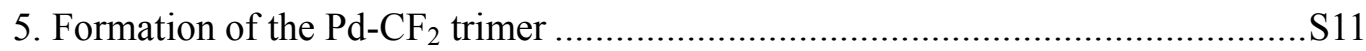

6. The observation of $\left[\left(\mathrm{Ph}_{3} \mathrm{P}\right)_{2} \mathrm{PdCF}_{2} \mathrm{H}\right]^{+}$complex........................................ 19

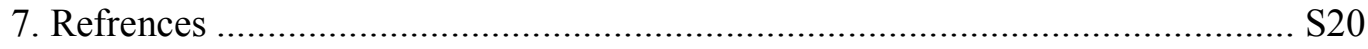

8. Copies of ${ }^{1} \mathrm{H}$ NMR, ${ }^{19} \mathrm{~F}$ NMR, ${ }^{13} \mathrm{C}$ NMR and ${ }^{31} \mathrm{P}$ NMR spectra ..................... S2 1 


\section{General information}

Solvents and reagents were purchased from commercial sources and used as received unless otherwise noted. The solvent $p$-xylene was distilled from $\mathrm{CaH}_{2} .{ }^{1} \mathrm{H}$, ${ }^{13} \mathrm{C},{ }^{19} \mathrm{~F}$, and ${ }^{31} \mathrm{P}$ NMR spectra were detected on a $500 \mathrm{MHz}, 400 \mathrm{MHz}$ or $300 \mathrm{MHz}$

NMR spectrometer. Data for ${ }^{1} \mathrm{H},{ }^{13} \mathrm{C},{ }^{19} \mathrm{~F}$ and ${ }^{31} \mathrm{P}$ NMR were recorded as follows: chemical shift $(\delta, \mathrm{ppm})$, multiplicity $(\mathrm{s}=$ singlet, $\mathrm{d}=$ doublet, $\mathrm{t}=$ triplet, $\mathrm{m}=$ multiplet, $\mathrm{q}=$ quartet, $\mathrm{br}=$ broad, coupling constant (s) in Hz). Mass spectra were obtained on a GC-MS. High resolution mass data were recorded on a high resolution mass spectrometer in the EI or ESI mode. Substrates 10 and 1r were commercially available. Substrates $\mathbf{1 p}, \mathbf{1 q}, \mathbf{1 s}$ and $\mathbf{1 t}$ were prepared according to the literature. ${ }^{1}$

\section{Procedure for the preparation of substrate $1 \mathrm{t}$}

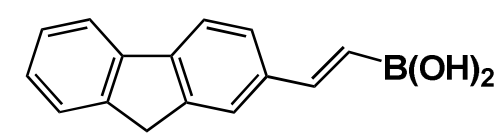

2-ethynyl-9H-fluorene (177mg, $0.93 \mathrm{mmol}$ ) and catecholborane (1 $\mathrm{M}$ in THF, $2 \mathrm{~mL}$ ) was stirred vigorously at $65{ }^{\circ} \mathrm{C}$ for $4 \mathrm{hr}$ under $\mathrm{N}_{2}$ atmosphere. The solvent was removed by concentration and then $\mathrm{H}_{2} \mathrm{O}(3 \mathrm{~mL})$ was added. The resulting mixture was stirred at $80{ }^{\circ} \mathrm{C}$ for $1 \mathrm{hr}$. A white precipitate was formed upon cooling, and was collected by filtration. Recrystallization of this solid from $\mathrm{THF} /$ hexane gave the pure product (1t). Yellow solid. $100.3 \mathrm{mg}, 46 \% ;{ }^{1} \mathrm{H}$ NMR (400 MHz, DMSO- $\left.d_{6}\right) \delta 7.90-$ 7.87 (m, 2H), 7.79 (brs, 2H), $7.70(\mathrm{~s}, 1 \mathrm{H}), 7.59$ (d, $J=7.7 \mathrm{~Hz}, 1 \mathrm{H}), 7.50$ (d, $J=7.7$ $\mathrm{Hz}, 1 \mathrm{H}), 7.42-7.29(\mathrm{~m}, 3 \mathrm{H}), 6.17(\mathrm{~d}, J=18.4 \mathrm{~Hz}, 1 \mathrm{H}), 3.94(\mathrm{~s}, 2 \mathrm{H}) .{ }^{13} \mathrm{C}$ NMR $(101$ MHz, DMSO- $\left.d_{6}\right) \delta 146.63$ (s), 144.02 (s), 143.91 (s), 141.98 (s), 141.19 (s), 136.91 (s), 127.41 (s), 127.29 (s), 126.34 (s), 125.63 (s), 123.57 (s), 123.05 (br), 120.65 (s), 120.61 (s), 36.81 (s). IR (neat) $v=3345,2922,2852,1685,1621,1568,1466,1455$, $1426,1375,1301,1246,1221,1199,1155,1044,986,949,882,817,765,732,648$, 571, 555, 516, $418 \mathrm{~cm}^{-1}$. HRMS (ESI) calcd. for $\mathrm{C}_{15} \mathrm{H}_{12} \mathrm{O}_{2}{ }^{10} \mathrm{~B}[\mathrm{M}-\mathrm{H}]^{+}: 234.0961$. Found: 234.0971. 


\section{Screening reaction conditions for $\mathrm{CF}_{2}$-transfer reaction}

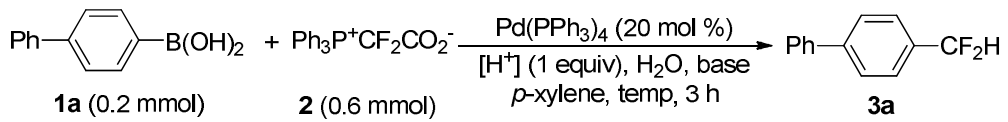

\begin{tabular}{|c|c|c|c|c|c|}
\hline entry & {$\left[\mathrm{H}^{+}\right]$} & $\begin{array}{l}\mathrm{H}_{2} \mathrm{O} \\
\text { (equiv) }\end{array}$ & $\begin{array}{l}\text { base } \\
\text { (equiv) }\end{array}$ & $\begin{array}{l}\text { temp } \\
\left({ }^{\circ} \mathrm{C}\right)\end{array}$ & $\begin{array}{l}\text { yield } \\
(\%)^{a}\end{array}$ \\
\hline 1 & - & - & $\mathrm{K}_{2} \mathrm{CO}_{3}(2.5)$ & 70 & trace \\
\hline 2 & $\mathrm{H}_{2} \mathrm{O}$ & - & $\mathrm{K}_{2} \mathrm{CO}_{3}(2.5)$ & 70 & trace \\
\hline 3 & $\mathrm{CH}_{3} \mathrm{COCH}_{2} \mathrm{CO}_{2} \mathrm{Et}$ & - & $\mathrm{K}_{2} \mathrm{CO}_{3}(2.5)$ & 70 & trace \\
\hline 4 & $\mathrm{CH}_{2}\left(\mathrm{CO}_{2} \mathrm{CH}_{3}\right)_{2}$ & - & $\mathrm{K}_{2} \mathrm{CO}_{3}(2.5)$ & 70 & trace \\
\hline 5 & $\mathrm{HCO}_{2} \mathrm{H}$ & - & $\mathrm{K}_{2} \mathrm{CO}_{3}(2.5)$ & 70 & 5 \\
\hline 6 & $\mathrm{CH}_{3} \mathrm{COCO}_{2} \mathrm{H}$ & - & $\mathrm{K}_{2} \mathrm{CO}_{3}(2.5)$ & 70 & 13 \\
\hline 7 & dione $^{b}$ & - & $\mathrm{K}_{2} \mathrm{CO}_{3}(2.5)$ & 70 & 27 \\
\hline 8 & dione & 2.5 & $\mathrm{~K}_{2} \mathrm{CO}_{3}(2.5)$ & 70 & 42 \\
\hline $9^{c}$ & dione & 2.5 & $\mathrm{~K}_{2} \mathrm{CO}_{3}(2.5)$ & 70 & 8 \\
\hline $10^{d}$ & dione & 2.5 & $\mathrm{~K}_{2} \mathrm{CO}_{3}(2.5)$ & 70 & 20 \\
\hline $11^{e}$ & dione & 2.5 & $\mathrm{~K}_{2} \mathrm{CO}_{3}(2.5)$ & 70 & trace \\
\hline $12^{f}$ & dione & 2.5 & $\mathrm{~K}_{2} \mathrm{CO}_{3}(2.5)$ & 70 & 5 \\
\hline $13^{g}$ & dione & 2.5 & $\mathrm{~K}_{2} \mathrm{CO}_{3}(2.5)$ & 70 & 0 \\
\hline $14^{h}$ & dione & 2.5 & $\mathrm{~K}_{2} \mathrm{CO}_{3}(2.5)$ & 70 & 12 \\
\hline 15 & dione & 2.5 & $\mathrm{KOH}(2.5)$ & 70 & 33 \\
\hline 16 & dione & 2.5 & $\mathrm{CsF}(2.5)$ & 70 & 14 \\
\hline 17 & dione & 2.5 & $\mathrm{Ca}(\mathrm{OH})_{2}(2.5)$ & 70 & 48 \\
\hline 18 & dione & 2.5 & $\mathrm{Ca}(\mathrm{OH})_{2}(4.0)$ & 70 & 61 \\
\hline 19 & dione & 2.5 & $\mathrm{Ca}(\mathrm{OH})_{2}(4.0)$ & 60 & 45 \\
\hline 20 & dione & 2.5 & $\mathrm{Ca}(\mathrm{OH})_{2}(4.0)$ & 80 & 67 \\
\hline 21 & dione & 2.5 & $\mathrm{Ca}(\mathrm{OH})_{2}(4.0)$ & 90 & 74 \\
\hline 22 & dione & 2.5 & $\mathrm{Ca}(\mathrm{OH})_{2}(4.0)$ & 100 & 62 \\
\hline $23^{i}$ & dione & 2.5 & $\mathrm{Ca}(\mathrm{OH})_{2}(4.0)$ & 90 & 82 \\
\hline $24^{j}$ & dione & 2.5 & $\mathrm{Ca}(\mathrm{OH})_{2}(4.0)$ & 90 & 90 \\
\hline $25^{k}$ & dione & 2.5 & $\mathrm{Ca}(\mathrm{OH})_{2}(4.0)$ & 90 & 26 \\
\hline $26^{l}$ & dione & 2.5 & $\mathrm{Ca}(\mathrm{OH})_{2}(4.0)$ & 90 & 0 \\
\hline
\end{tabular}

${ }^{a}$ Determined by ${ }^{19} \mathrm{~F}$ NMR; ${ }^{b}$ dione $=1,3$-cyclopentadione; ${ }^{c} \mathrm{Pd}_{2}(\mathrm{dba})_{3}(10 \mathrm{~mol} \%)$ instead of $\operatorname{Pd}\left(\mathrm{PPh}_{3}\right)_{4}(20 \mathrm{~mol} \%)$ was used as the catalyst; ${ }^{d} \mathrm{Pd}_{2}(\mathrm{dba})_{3}(10 \mathrm{~mol} \%) / \mathrm{XPhos}(20 \mathrm{~mol} \%)$ instead 
of $\mathrm{Pd}\left(\mathrm{PPh}_{3}\right)_{4}(20 \mathrm{~mol} \%)$ were used as the catalyst/ligand; ${ }^{e} \mathrm{Pd}_{2}(\mathrm{dba})_{3}(10 \mathrm{~mol} \%) / \mathrm{Xantphos}(20$ mol \% ) instead of $\mathrm{Pd}\left(\mathrm{PPh}_{3}\right)_{4}(20 \mathrm{~mol} \%)$ were used as the catalyst/ligand; ${ }^{f} \mathrm{Pd}\left(\mathrm{Ph}_{3} \mathrm{P}\right)_{2} \mathrm{Cl}_{2}(20$ mol \% ) instead of $\mathrm{Pd}\left(\mathrm{PPh}_{3}\right)_{4}(20 \mathrm{~mol} \%)$ was used as the catalyst; ${ }^{g} \mathrm{DMF}$ was used as the solvent;

${ }^{h}$ 1,4-dioxane was used as the solvent; ${ }^{i} 4$ equiv of PDFA was used; ${ }^{j} 5$ equiv of PDFA was used. ${ }^{k} 10$ $\mathrm{mol} \%$ of $\mathrm{Pd}\left(\mathrm{PPh}_{3}\right)_{4}$ was uesed. ${ }^{l}$ no catalyst was used.

\section{General procedure for the Pd-catalyzed difluorocarbene transfer reaction}

A dried Schlenk tube was charged with arylboronic acid or vinylboronic acid (0.2 $\mathrm{mmol}), \mathrm{Ph}_{3} \mathrm{P}^{+} \mathrm{CF}_{2} \mathrm{CO}_{2}^{-}$(356 mg, $\left.1.0 \mathrm{mmol}\right), 1,3-C y c l o p e n d i o n e ~(19.6 \mathrm{mg}, 0.2 \mathrm{mmol})$, $\mathrm{Ca}(\mathrm{OH})_{2}(59.3 \mathrm{mg}, 0.8 \mathrm{mmol}), \mathrm{Pd}\left(\mathrm{PPh}_{3}\right)_{4}(46.2 \mathrm{mg}, 0.04 \mathrm{mmol}), \mathrm{H}_{2} \mathrm{O}(9 \mathrm{mg}, 0.5$ mmol) and $p$-xylene $(2 \mathrm{~mL})$. The mixture was stirred at $90{ }^{\circ} \mathrm{C}$ for $3 \mathrm{~h}$ under $\mathrm{N}_{2}$ atmosphere. After being cooled to room temperature, the mixture was subjected to flash column chromatography (petroleum ether / dichloromethane) to afford the pure product.

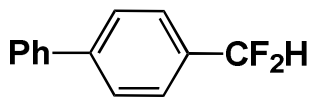

\section{4-(difluoromethyl)-1,1'-biphenyl (3a) ${ }^{2}$ :}

White solid. $34.3 \mathrm{mg}, 84 \%$; ${ }^{1} \mathrm{H}$ NMR $\left(400 \mathrm{MHz}, \mathrm{CDCl}_{3}\right) \delta 7.65(\mathrm{~d}, J=8.1 \mathrm{~Hz}$, 2H), $7.59-7.55(\mathrm{~m}, 4 \mathrm{H}), 7.47-7.43(\mathrm{~m}, 2 \mathrm{H}), 7.39-7.35(\mathrm{~m}, 1 \mathrm{H}), 6.68(\mathrm{t}, J=56.5$ $\mathrm{Hz}, 1 \mathrm{H}) .{ }^{19} \mathrm{~F}$ NMR $\left(376 \mathrm{MHz}, \mathrm{CDCl}_{3}\right) \delta-110.34$ (d, $\left.J=56.5 \mathrm{~Hz}, 2 \mathrm{~F}\right) . \mathrm{GC}-\mathrm{MS}(\mathrm{EI})$ calcd. for $\mathrm{C}_{13} \mathrm{H}_{10} \mathrm{~F}_{2}[\mathrm{M}]^{+}: 204.1$. Found: 204.1.

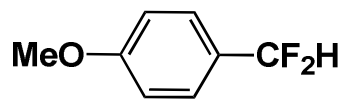

1-(difluoromethyl)-4-methoxybenzene $(3 b)^{3}$ :

Colourless liquid. $26.2 \mathrm{mg}, 83 \%$; ${ }^{1} \mathrm{H}$ NMR $\left(400 \mathrm{MHz}, \mathrm{CDCl}_{3}\right) \delta 7.44(\mathrm{~d}, J=8.6$ $\mathrm{Hz}, 2 \mathrm{H}), 6.95(\mathrm{~d}, J=8.6 \mathrm{~Hz}, 2 \mathrm{H}), 6.60(\mathrm{t}, J=56.8 \mathrm{~Hz}, 1 \mathrm{H}), 3.84(\mathrm{~s}, 3 \mathrm{H}) .{ }^{19} \mathrm{~F}$ NMR $\left(376 \mathrm{MHz}, \mathrm{CDCl}_{3}\right) \delta-108.28(\mathrm{~d}, J=56.8 \mathrm{~Hz}, 2 \mathrm{~F})$. GC-MS(EI) calcd. for $\mathrm{C}_{8} \mathrm{H}_{8} \mathrm{~F}_{2} \mathrm{O}$ $[\mathrm{M}]^{+}:$158.1. Found: 158.1. 


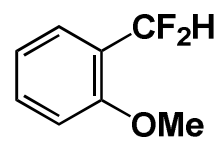

1-(difluoromethyl)-2-methoxybenzene $(3 c)^{2}$ :

Colourless liquid. $12.7 \mathrm{mg}, 40 \%$; ${ }^{1} \mathrm{H}$ NMR $\left(400 \mathrm{MHz}, \mathrm{CDCl}_{3}\right) \delta 7.56(\mathrm{~d}, J=7.6$ $\mathrm{Hz}, 1 \mathrm{H}), 7.47-7.40(\mathrm{~m}, 1 \mathrm{H}), 7.11-6.80(\mathrm{~m}, 3 \mathrm{H}), 3.87(\mathrm{~s}, 3 \mathrm{H}) .{ }^{19} \mathrm{~F}$ NMR $(376 \mathrm{MHz}$, $\left.\mathrm{CDCl}_{3}\right) \delta-115.44(\mathrm{~d}, J=55.7 \mathrm{~Hz}, 2 \mathrm{~F})$. GC-MS(EI) calcd. for $\mathrm{C}_{8} \mathrm{H}_{8} \mathrm{~F}_{2} \mathrm{O}[\mathrm{M}]^{+}:$: 58.1 .

Found: 158.1.

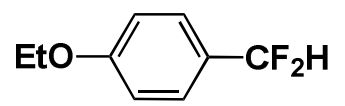

\section{1-(difluoromethyl)-4-ethoxybenzene (3d):}

Colourless liquid. $23.5 \mathrm{mg}, 68 \%$; ${ }^{1} \mathrm{H}$ NMR $\left(400 \mathrm{MHz}, \mathrm{CDCl}_{3}\right) \delta 7.42(\mathrm{~d}, J=8.3 \mathrm{~Hz}$, 2H), 6.93 (d, $J=8.5 \mathrm{~Hz}, 2 \mathrm{H}), 6.59$ (t, $J=56.8 \mathrm{~Hz}, 1 \mathrm{H}), 4.06$ (q, $J=7.0 \mathrm{~Hz}, 2 \mathrm{H}), 1.43$ $(\mathrm{t}, J=7.0 \mathrm{~Hz}, 3 \mathrm{H}) .{ }^{19} \mathrm{~F}$ NMR $\left(376 \mathrm{MHz}, \mathrm{CDCl}_{3}\right) \delta-108.22(\mathrm{~d}, J=56.8 \mathrm{~Hz}, 2 \mathrm{~F}) .{ }^{13} \mathrm{C}$ NMR (101 MHz, $\left.\mathrm{CDCl}_{3}\right) \delta 160.76(\mathrm{t}, J=1.6 \mathrm{~Hz}), 127.08$ (t, $\left.J=5.9 \mathrm{~Hz}\right), 126.59$ (t, $J$ $=22.6 \mathrm{~Hz}), 114.95(\mathrm{t}, J=237.3 \mathrm{~Hz}), 114.51(\mathrm{~s}), 63.61(\mathrm{~s}), 14.70(\mathrm{~s})$. IR (neat) $v=$ 3676, 2984, 2934, 2557, 2364, 1897, 1617, 1589, 1519, 1479, 1433, 1384, 1307, 1254, $1226,1176,1114,1071,1045,1020,940,923,859,829,788,653,639,626,552 \mathrm{~cm}^{-1}$. HRMS (EI) calcd. for $\mathrm{C}_{9} \mathrm{H}_{10} \mathrm{~F}_{2} \mathrm{O}[\mathrm{M}]^{+}:$172.0700. Found: 172.0696 .

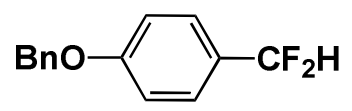

1-(benzyloxy)-4-(difluoromethyl)benzene $(3 \mathrm{e})^{2}$ :

White solid. $37.3 \mathrm{mg}, 80 \% ;{ }^{1} \mathrm{H}$ NMR (400 MHz, $\left.\mathrm{CDCl}_{3}\right) \delta 7.44-7.30(\mathrm{~m}, 7 \mathrm{H})$, $7.02(\mathrm{~d}, J=8.5 \mathrm{~Hz}, 2 \mathrm{H}), 6.58(\mathrm{t}, J=56.7 \mathrm{~Hz}, 1 \mathrm{H}), 5.08(\mathrm{~s}, 2 \mathrm{H}) .{ }^{19} \mathrm{~F}$ NMR $(376 \mathrm{MHz}$, $\left.\mathrm{CDCl}_{3}\right) \delta-108.37(\mathrm{~d}, J=56.7 \mathrm{~Hz}, 2 \mathrm{~F})$. GC-MS(EI) calcd. for $\mathrm{C}_{14} \mathrm{H}_{12} \mathrm{~F}_{2} \mathrm{O}[\mathrm{M}]^{+}: 234.1$. Found: 234.1 . 


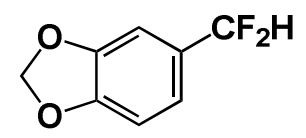

5-(difluoromethyl)benzo[d] $[1,3]$ dioxole $(3 f)^{3}$ :

Colourless liquid. $23.5 \mathrm{mg}, 68 \%$; ${ }^{1} \mathrm{H}$ NMR (400 MHz, $\left.\mathrm{CDCl}_{3}\right) \delta 6.97$ (d, $J=8.2$ $\mathrm{Hz}, 2 \mathrm{H}), 6.84(\mathrm{~d}, J=8.2 \mathrm{~Hz}, 1 \mathrm{H}), 6.54(\mathrm{t}, J=56.6 \mathrm{~Hz}, 1 \mathrm{H}), 6.01(\mathrm{~s}, 2 \mathrm{H}) .{ }^{19} \mathrm{~F}$ NMR $\left(376 \mathrm{MHz}, \mathrm{CDCl}_{3}\right) \delta-107.96(\mathrm{~d}, J=56.6 \mathrm{~Hz}, 2 \mathrm{~F})$. GC-MS(EI) calcd. for $\mathrm{C}_{8} \mathrm{H}_{6} \mathrm{~F}_{2} \mathrm{O}_{2}$ $[\mathrm{M}]^{+}:$172.0. Found: 172.0 .

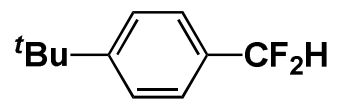

\section{1-(tert-butyl)-4-(difluoromethyl)benzene (3g) ${ }^{2}$ :}

Colourless liquid. $29.5 \mathrm{mg}, 80 \% ;{ }^{1} \mathrm{H}$ NMR $\left(400 \mathrm{MHz}, \mathrm{CDCl}_{3}\right) \delta 7.48(\mathrm{~d}, J=8.6$ $\mathrm{Hz}, 2 \mathrm{H}), 7.44(\mathrm{~d}, J=8.6 \mathrm{~Hz}, 2 \mathrm{H}), 6.63(\mathrm{t}, J=56.6 \mathrm{~Hz}, 1 \mathrm{H}), 1.34(\mathrm{~s}, 9 \mathrm{H}) .{ }^{19} \mathrm{~F}$ NMR (376 MHz, $\mathrm{CDCl}_{3}$ ) $\delta-109.95$ (d, $\left.J=56.6 \mathrm{~Hz}, 2 \mathrm{~F}\right)$. GC-MS(EI) calcd. for $\mathrm{C}_{8} \mathrm{H}_{6} \mathrm{~F}_{2} \mathrm{O}_{2}$ $[\mathrm{M}]^{+}:$172.0. Found: 172.0 .

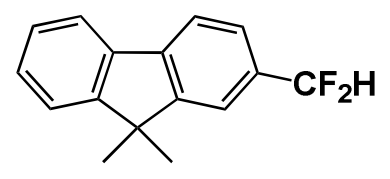

\section{2-(difluoromethyl)-9,9-dimethyl-9H-fluorene (3h)}

Colourless liquid. $31.6 \mathrm{mg}, 65 \% ;{ }^{1} \mathrm{H}$ NMR (400 MHz, $\left.\mathrm{CDCl}_{3}\right) \delta 7.79-7.70(\mathrm{~m}$, 2H), $7.57(\mathrm{~s}, 1 \mathrm{H}), 7.48-7.41(\mathrm{~m}, 2 \mathrm{H}), 7.38-7.32(\mathrm{~m}, 2 \mathrm{H}), 6.70(\mathrm{t}, J=56.7 \mathrm{~Hz}, 1 \mathrm{H})$, 1.49 (s, 6H). ${ }^{19} \mathrm{~F}$ NMR $\left(376 \mathrm{MHz}, \mathrm{CDCl}_{3}\right) \delta-108.97$ (d, $\left.J=56.7 \mathrm{~Hz}, 2 \mathrm{~F}\right) .{ }^{13} \mathrm{C}$ NMR (101 MHz, $\left.\mathrm{CDCl}_{3}\right) \delta 154.04$ (s), 154.03 (s), 141.78 (t, $\left.J=2.0 \mathrm{~Hz}\right), 138.12$ (s), 133.11 (t, $J=22.0 \mathrm{~Hz}), 128.09$ (s), 127.17 (s), 124.76 (t, $J=6.3 \mathrm{~Hz}), 122.75$ (s), 120.52 (s), 120.10 (s), 119.83 (t, $J=5.9 \mathrm{~Hz}), 115.23$ (t, $J=238.4 \mathrm{~Hz}), 47.03$ (s), 27.00 (s). IR (neat) $v=3064,3017,2963,2925,2862,1618,1588,1474,1458,1450,1427,1371$, $1342,1302,1280,1214,1193,1157,1140,1120,1067,1024,941,896,836,784,758$, 
738, 712, 569, 469, $443 \mathrm{~cm}^{-1}$. HRMS (EI) calcd. for $\mathrm{C}_{16} \mathrm{H}_{14} \mathrm{~F}_{2}[\mathrm{M}]^{+}:$244.1064. Found: 244.1059.

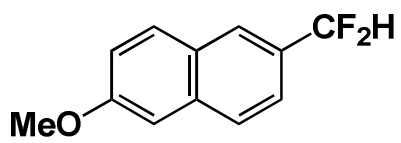

\section{2-(difluoromethyl)-6-methoxynaphthalene (3i):}

White solid. M.p. $75-76{ }^{\circ} \mathrm{C} .32 .9 \mathrm{mg}, 79 \%$; ${ }^{1} \mathrm{H}$ NMR $\left(400 \mathrm{MHz}, \mathrm{CDCl}_{3}\right) \delta 7.87$ (s, 1H), $7.80-7.76(\mathrm{~m}, 2 \mathrm{H}), 7.55(\mathrm{~d}, J=8.5 \mathrm{~Hz}, 1 \mathrm{H}), 7.22-7.12(\mathrm{~m}, 2 \mathrm{H}), 6.76(\mathrm{t}, J=$ $56.5 \mathrm{~Hz}, 1 \mathrm{H}), 3.92(\mathrm{~s}, 3 \mathrm{H}) .{ }^{19} \mathrm{~F}$ NMR $\left(376 \mathrm{MHz}, \mathrm{CDCl}_{3}\right) \delta-109.09(\mathrm{~d}, J=56.5 \mathrm{~Hz}$, 2F). ${ }^{13} \mathrm{C}$ NMR (101 MHz, $\left.\mathrm{CDCl}_{3}\right) \delta 158.82$ (s), 135.78 (s), 130.00 (s), 129.48 (t, $J=$ $22.3 \mathrm{~Hz}), 127.98$ (s), $127.62(\mathrm{~s}), 125.68$ (t, $J=7.5 \mathrm{~Hz}), 122.63$ (t, $J=4.8 \mathrm{~Hz}), 119.72$ (s), 115.25 (t, $J=237.9 \mathrm{~Hz}), 105.81$ (s), 55.36 (s). IR (neat) $v=2976,2946,2850$, $1932,1802,1634,1613,1508,1492,1462,1452,1441,1428,1393,1358,1332,1276$, $1245,1196,1179,1164,1124,1087,1026,925,904,858,837,826,782,722,678$, 639, 513, 481, 471, $458 \mathrm{~cm}^{-1}$. HRMS (EI) calcd. for $\mathrm{C}_{12} \mathrm{H}_{10} \mathrm{~F}_{2} \mathrm{O}[\mathrm{M}]^{+}: 208.0700$. Found: 208.0698.

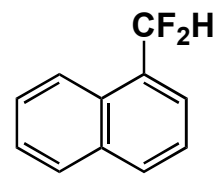

\section{1-(difluoromethyl)naphthalene (3k) ${ }^{3}$ :}

Colourless liquid. $27.9 \mathrm{mg}, 78 \%$; ${ }^{1} \mathrm{H}$ NMR $\left(400 \mathrm{MHz}, \mathrm{CDCl}_{3}\right) \delta 8.16(\mathrm{~d}, J=8.3$ $\mathrm{Hz}, 1 \mathrm{H}), 7.96-7.88(\mathrm{~m}, 2 \mathrm{H}), 7.68(\mathrm{~d}, J=7.1 \mathrm{~Hz}, 1 \mathrm{H}), 7.62-7.45(\mathrm{~m}, 3 \mathrm{H}), 7.12$ (t, $J$ $=55.2 \mathrm{~Hz}, 1 \mathrm{H}) .{ }^{19} \mathrm{~F}$ NMR $\left(376 \mathrm{MHz}, \mathrm{CDCl}_{3}\right) \delta-110.90(\mathrm{~d}, J=55.2 \mathrm{~Hz}, 2 \mathrm{~F})$. GC-MS(EI) calcd. for $\mathrm{C}_{11} \mathrm{H}_{8} \mathrm{~F}_{2}[\mathrm{M}]^{+}$: 178.1. Found: 178.1 . 


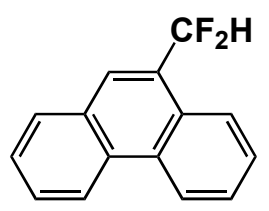

\section{9-(difluoromethyl)phenanthrene (3I) ${ }^{3}$ :}

White solid. $38.1 \mathrm{mg}, 84 \%$; ${ }^{1} \mathrm{H}$ NMR $\left(400 \mathrm{MHz}, \mathrm{CDCl}_{3}\right) \delta 8.71(\mathrm{~d}, J=7.8 \mathrm{~Hz}$, $1 \mathrm{H}), 8.65(\mathrm{~d}, J=8.3 \mathrm{~Hz}, 1 \mathrm{H}), 8.20(\mathrm{~d}, J=8.3 \mathrm{~Hz}, 1 \mathrm{H}), 7.99-7.87$ (m, 2H), $7.73-$ $7.58(\mathrm{~m}, 4 \mathrm{H}), 7.13(\mathrm{t}, J=55.1 \mathrm{~Hz}, 1 \mathrm{H}) .{ }^{19} \mathrm{~F}$ NMR $\left(376 \mathrm{MHz}, \mathrm{CDCl}_{3}\right) \delta-111.78(\mathrm{~d}, J=$ $55.0 \mathrm{~Hz}, 2 \mathrm{~F})$. GC-MS(EI) calcd. for $\mathrm{C}_{15} \mathrm{H}_{10} \mathrm{~F}_{2}[\mathrm{M}]^{+}$: 228.1. Found: 228.1 .

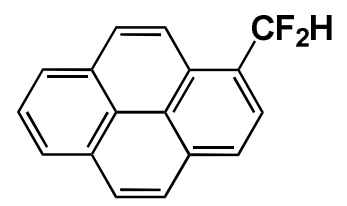

\section{1-(difluoromethyl)pyrene (3m):}

Yellow solid. M.p. $98-99{ }^{\circ} \mathrm{C} .43 .2 \mathrm{mg}, 86 \% ;{ }^{1} \mathrm{H}$ NMR $\left(400 \mathrm{MHz}, \mathrm{CDCl}_{3}\right) \delta$ $8.29(\mathrm{~d}, J=9.2 \mathrm{~Hz}, 1 \mathrm{H}), 8.19-7.95(\mathrm{~m}, 8 \mathrm{H}), 7.41(\mathrm{t}, J=55.4 \mathrm{~Hz}, 1 \mathrm{H}) .{ }^{19} \mathrm{~F}$ NMR $(376$ $\left.\mathrm{MHz}, \mathrm{CDCl}_{3}\right) \delta-108.86(\mathrm{~d}, J=55.4 \mathrm{~Hz}, 2 \mathrm{~F}) .{ }^{13} \mathrm{C} \mathrm{NMR}\left(101 \mathrm{MHz}, \mathrm{CDCl}_{3}\right) \delta 133.12(\mathrm{t}$, $J=1.7 \mathrm{~Hz}$ ), 131.08 (s), 130.45 (s), 128.95 (s), 128.86 (s), 128.48 (t, $J=3.7 \mathrm{~Hz}$ ), $127.20(\mathrm{t}, J=1.0 \mathrm{~Hz}), 126.42(\mathrm{t}, J=21.1 \mathrm{~Hz}), 126.39$ (s), $126.10(\mathrm{~s}), 126.01(\mathrm{~s})$, 124.79 (s), 124.41 (s), 124.38 (s), 123.59 (t, $J=7.9 \mathrm{~Hz}), 122.28$ (s), 115.36 (t, $J=$ $238.2 \mathrm{~Hz}$ ). IR (neat) $v=3042,1916,1742,1628,1596,1513,1418,1401,1383$, $1341,1306,1250,1238,1190,1177,1166,1140,1117,1081,1015,970,905,884$, $847,829,799,781,752,713,692,681,641,539,505 \mathrm{~cm}^{-1}$. HRMS (EI) calcd. for $\mathrm{C}_{17} \mathrm{H}_{10} \mathrm{~F}_{2}[\mathrm{M}]^{+}:$252.0751. Found: 252.0755.

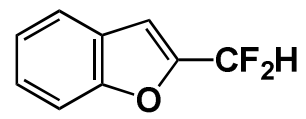

\section{2-(difluoromethyl)benzofuran (3n) ${ }^{4}$ :}


White solid. $22.8 \mathrm{mg}, 68 \%$; ${ }^{1} \mathrm{H}$ NMR $\left(400 \mathrm{MHz}, \mathrm{CDCl}_{3}\right) \delta 7.63(\mathrm{~d}, J=7.8 \mathrm{~Hz}$, 1H), $7.53(\mathrm{~d}, J=8.2 \mathrm{~Hz}, 1 \mathrm{H}), 7.37$ (t, $J=7.6 \mathrm{~Hz}, 1 \mathrm{H}), 7.28$ (t, $J=7.5 \mathrm{~Hz}, 1 \mathrm{H}), 7.01$ (s, $1 \mathrm{H}), 6.74(\mathrm{t}, J=54.2 \mathrm{~Hz}, 1 \mathrm{H}) .{ }^{19} \mathrm{~F}$ NMR $\left(376 \mathrm{MHz}, \mathrm{CDCl}_{3}\right) \delta-116.61(\mathrm{dd}, J=54.2$, $1.7 \mathrm{~Hz}, 2 \mathrm{~F})$. GC-MS(EI) calcd. for $\mathrm{C}_{9} \mathrm{H}_{6} \mathrm{~F}_{2} \mathrm{O}[\mathrm{M}]^{+}$: 168.0. Found: 168.0.

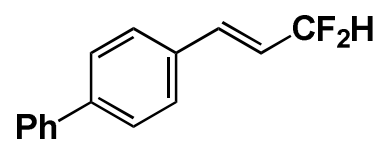

\section{(E)-4-(3,3-difluoroprop-1-en-1-yl)-1,1'-biphenyl (3o):}

White solid. M.p. $126-127{ }^{\circ} \mathrm{C} .34 .3 \mathrm{mg}, 74 \% ;{ }^{1} \mathrm{H}$ NMR $\left(400 \mathrm{MHz}, \mathrm{CDCl}_{3}\right) \delta$ $7.59(\mathrm{~d}, J=8.0 \mathrm{~Hz}, 4 \mathrm{H}), 7.51-7.41(\mathrm{~m}, 4 \mathrm{H}), 7.35(\mathrm{t}, J=7.3 \mathrm{~Hz}, 1 \mathrm{H}), 6.93-6.85(\mathrm{~m}$, 1H), $6.41-6.09(\mathrm{~m}, 2 \mathrm{H}) .{ }^{19} \mathrm{~F}$ NMR $\left(376 \mathrm{MHz}, \mathrm{CDCl}_{3}\right) \delta-109.45--109.65(\mathrm{~m}, 2 \mathrm{~F})$. ${ }^{13} \mathrm{C}$ NMR (101 MHz, $\left.\mathrm{CDCl}_{3}\right) \delta 142.17$ (s), 140.23 (s), 136.63 (t, $\left.J=12.1 \mathrm{~Hz}\right), 133.36$ (s), 128.87 (s), 127.70 (s), 127.46 (s), 127.01 (s), 120.86 (t, $J=23.9 \mathrm{~Hz}), 115.41$ (t, $J$ $=233.7 \mathrm{~Hz}$ ). IR (neat) $v=1660,1488,1412,1393,1140,1062,1003,963,862,820$, 763, 689, $518 \mathrm{~cm}^{-1}$. HRMS (EI) calcd. for $\mathrm{C}_{15} \mathrm{H}_{12} \mathrm{~F}_{2}[\mathrm{M}]^{+}: 230.0907$. Found: 230.0910 .

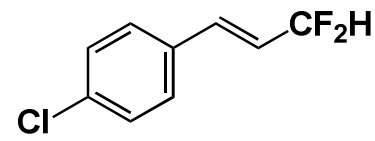

\section{(E)-1-chloro-4-(3,3-difluoroprop-1-en-1-yl)benzene (3p) ${ }^{5}$ :}

Colourless liquid. $23.5 \mathrm{mg}, 62 \% ;{ }^{1} \mathrm{H}$ NMR $\left(400 \mathrm{MHz}, \mathrm{CDCl}_{3}\right) \delta 7.38-7.32(\mathrm{~m}$, 4H), $6.87-6.80(\mathrm{~m}, 1 \mathrm{H}), 6.44-6.04(\mathrm{~m}, 2 \mathrm{H}) .{ }^{19} \mathrm{~F}$ NMR $\left(376 \mathrm{MHz}, \mathrm{CDCl}_{3}\right) \delta-110.17$ (ddd, $J=56.5,7.7,3.6 \mathrm{~Hz}, 2 \mathrm{~F}$ ). GC-MS(EI) calcd. for $\mathrm{C}_{9} \mathrm{H}_{7} \mathrm{ClF}_{2}[\mathrm{M}]^{+}: 188.0$. Found: 188.0 .

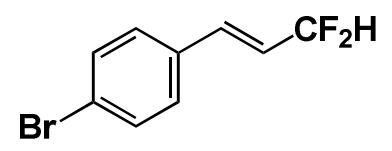

\section{(E)-1-bromo-4-(3,3-difluoroprop-1-en-1-yl)benzene $(3 q)^{5}$ :}


White solid. $30.1 \mathrm{mg}, 65 \%$; ${ }^{1} \mathrm{H}$ NMR $\left(400 \mathrm{MHz}, \mathrm{CDCl}_{3}\right) \delta 7.48(\mathrm{~d}, J=8.3 \mathrm{~Hz}$, 2H), $7.28(\mathrm{~d}, J=8.3 \mathrm{~Hz}, 2 \mathrm{H}), 6.85-6.76(\mathrm{~m}, 1 \mathrm{H}), 6.38-6.05(\mathrm{~m}, 2 \mathrm{H}) .{ }^{19} \mathrm{~F}$ NMR $\left(376 \mathrm{MHz}, \mathrm{CDCl}_{3}\right) \delta-110.19-110.35(\mathrm{~m}, 2 \mathrm{~F})$. GC-MS(EI) calcd. for $\mathrm{C}_{9} \mathrm{H}_{7} \mathrm{BrF}_{2}[\mathrm{M}]^{+}$: 232.0. Found: 232.0 .

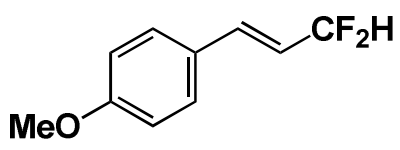

\section{(E)-1-(3,3-difluoroprop-1-en-1-yl)-4-methoxybenzene (3r) $)^{5}$ :}

White solid. $19.1 \mathrm{mg}, 52 \% ;{ }^{1} \mathrm{H}$ NMR $\left(400 \mathrm{MHz}, \mathrm{CDCl}_{3}\right) \delta 7.36(\mathrm{~d}, J=8.6 \mathrm{~Hz}$, 2H), $6.88(\mathrm{~d}, J=8.6 \mathrm{~Hz}, 2 \mathrm{H}), 6.83-6.76(\mathrm{~m}, 1 \mathrm{H}), 6.37-6.05(\mathrm{~m}, 2 \mathrm{H}), 3.81(\mathrm{~s}, 3 \mathrm{H})$. ${ }^{19} \mathrm{~F}$ NMR $\left(376 \mathrm{MHz}, \mathrm{CDCl}_{3}\right) \delta-108.58--108.81$ (m, 2F). GC-MS(EI) calcd. for $\mathrm{C}_{10} \mathrm{H}_{10} \mathrm{~F}_{2} \mathrm{O}[\mathrm{M}]^{+}:$184.1. Found: 184.1.

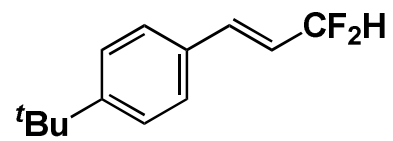

\section{(E)-1-(tert-butyl)-4-(3,3-difluoroprop-1-en-1-yl)benzene (3s) ${ }^{2}$ :}

White solid. $34.8 \mathrm{mg}, 83 \%$; ${ }^{1} \mathrm{H}$ NMR (400 MHz, $\left.\mathrm{CDCl}_{3}\right) \delta 7.42-7.35$ (m, 4H), $6.90-6.81(\mathrm{~m}, 1 \mathrm{H}), 6.39-6.08(\mathrm{~m}, 2 \mathrm{H}), 1.32(\mathrm{~s}, 9 \mathrm{H}) .{ }^{19} \mathrm{~F} \mathrm{NMR}\left(376 \mathrm{MHz}, \mathrm{CDCl}_{3}\right) \delta$ $-109.14--109.33(\mathrm{~m}, 2 \mathrm{~F})$. GC-MS(EI) calcd. for $\mathrm{C}_{13} \mathrm{H}_{16} \mathrm{~F}_{2}[\mathrm{M}]^{+}:$: 210.1. Found: 210.1.

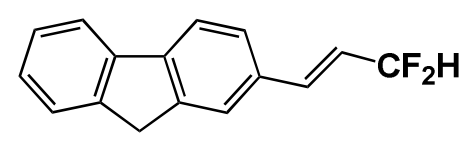

\section{(E)-2-(3,3-difluoroprop-1-en-1-yl)-9H-fluorene (3t):}

White solid. $31.1 \mathrm{mg}, 64 \%$; ${ }^{1} \mathrm{H}$ NMR (400 MHz, $\left.\mathrm{CDCl}_{3}\right) \delta 7.77-7.19(\mathrm{~m}, 2 \mathrm{H})$, $7.57(\mathrm{~s}, 1 \mathrm{H}), 7.52(\mathrm{~d}, J=7.4 \mathrm{~Hz}, 1 \mathrm{H}), 7.43-7.28(\mathrm{~m}, 3 \mathrm{H}), 6.95-6.84(\mathrm{~m}, 1 \mathrm{H}), 6.42$

$-6.08(\mathrm{~m}, 2 \mathrm{H}), 3.85(\mathrm{~s}, 2 \mathrm{H}) .{ }^{19} \mathrm{~F}$ NMR $\left(376 \mathrm{MHz}, \mathrm{CDCl}_{3}\right) \delta-108.94--109.15(\mathrm{~m}$, 2F). $\left.{ }^{13} \mathrm{C} \mathrm{NMR} \mathrm{(101} \mathrm{MHz,} \mathrm{CDCl}_{3}\right) \delta 143.80$ (s), 143.69 (s), 143.13 (s), 141.01 (s), 
137.47 (t, $J=12.2 \mathrm{~Hz}), 132.97$ (s), 127.29 (s), 126.97 (s), 126.51 (s), 125.15 (s), 123.66 (s), 120.23 (s), 120.17 (t, $J=24.0 \mathrm{~Hz}), 120.10$ (s), 115.67 (t, $J=233.5 \mathrm{~Hz}$ ), 36.81 (s). IR (neat) $v=3853,2999,1659,1456,1427,1387,1158,1138,1062,1014$, 998, 979, 970, 924, 879, 826, 768, 737, 525, 428, $415 \mathrm{~cm}^{-1}$. HRMS (EI) calcd. for $\mathrm{C}_{16} \mathrm{H}_{12} \mathrm{~F}_{2}[\mathrm{M}]^{+}:$242.0907. Found: 242.0906.

\section{Formation of the $\mathrm{Pd}-\mathrm{CF}_{2}$ trimer}

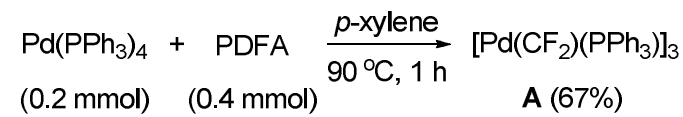
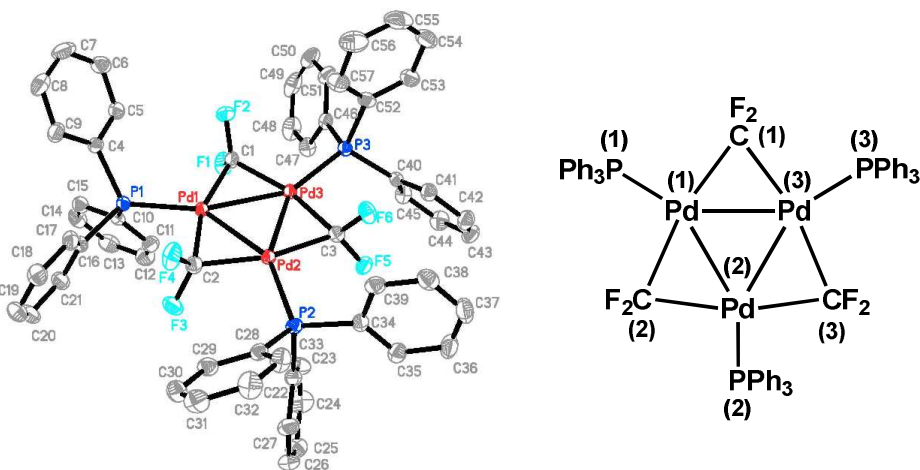

A dried Schlenk tube was charged with $\mathrm{Pd}\left(\mathrm{PPh}_{3}\right)_{4}(231 \mathrm{mg}, 0.2 \mathrm{mmol})$, $\mathrm{Ph}_{3} \mathrm{P}^{+} \mathrm{CF}_{2} \mathrm{CO}_{2}^{-}(143 \mathrm{mg}, 0.4 \mathrm{mmol})$ and $p$-xylene $(2 \mathrm{~mL})$. The resulting mixture was stirred at $90{ }^{\circ} \mathrm{C}$ for $1 \mathrm{~h}$ under $\mathrm{N}_{2}$ atmosphere. After the solvent was removed by concentration, the mixture was recrystallized from $n$-hexane / dichloromethane (20:1) to afford pure product as a dark red solid. ${ }^{1} \mathrm{H}$ NMR $\left(400 \mathrm{MHz}, \mathrm{CDCl}_{3}\right) \delta 7.41$ (br, $18 \mathrm{H}), 7.33-7.28(\mathrm{~m}, 27 \mathrm{H}) .{ }^{19} \mathrm{~F}$ NMR $\left(376 \mathrm{MHz}, \mathrm{CDCl}_{3}\right) \delta 84.68\left(\mathrm{AB}, \Delta v_{\mathrm{AB}}=14.4\right.$ $\left.\mathrm{Hz}, J_{\mathrm{AB}}=8.3 \mathrm{~Hz}, 6 \mathrm{~F}\right) .{ }^{31} \mathrm{P}$ NMR $\left(162 \mathrm{MHz}, \mathrm{CD}_{3} \mathrm{OD}\right) \delta 31.73$ (s, 3P). Anal. Calcd. for $\mathrm{C}_{57} \mathrm{H}_{45} \mathrm{~F}_{6} \mathrm{P}_{3} \mathrm{Pd}_{3}: \mathrm{C}, 54.50 ; \mathrm{H}, 3.61$. Found: $\mathrm{C}, 54.19 ; \mathrm{H}, 3.51$.

Bond lengths $[\AA]$ for Complex A:

$\begin{array}{ll}\operatorname{Pd}(1)-\mathrm{C}(2) & 2.081(3) \\ \operatorname{Pd}(1)-\mathrm{C}(1) & 2.082(3) \\ \operatorname{Pd}(1)-\mathrm{P}(1) & 2.3239(6) \\ \operatorname{Pd}(1)-\operatorname{Pd}(2) & 2.5891(3) \\ \operatorname{Pd}(1)-\operatorname{Pd}(3) & 2.5916(3)\end{array}$




\begin{tabular}{|c|c|}
\hline $\operatorname{Pd}(2)-C(2)$ & $2.051(3)$ \\
\hline $\operatorname{Pd}(2)-C(3)$ & $2.072(3)$ \\
\hline $\mathrm{Pd}(2)-\mathrm{P}(2)$ & $2.3173(6)$ \\
\hline $\operatorname{Pd}(2)-\operatorname{Pd}(3)$ & $2.5764(3)$ \\
\hline $\operatorname{Pd}(3)-C(1)$ & $2.058(2)$ \\
\hline $\operatorname{Pd}(3)-C(3)$ & $2.090(3)$ \\
\hline $\operatorname{Pd}(3)-P(3)$ & $2.3204(7)$ \\
\hline $\mathrm{P}(1)-\mathrm{C}(10)$ & $1.826(3)$ \\
\hline $\mathrm{P}(1)-\mathrm{C}(16)$ & $1.830(3)$ \\
\hline $\mathrm{P}(1)-\mathrm{C}(4)$ & $1.834(3)$ \\
\hline $\mathrm{P}(2)-\mathrm{C}(22)$ & $1.823(3)$ \\
\hline $\mathrm{P}(2)-\mathrm{C}(34)$ & $1.831(3)$ \\
\hline $\mathrm{P}(2)-\mathrm{C}(28)$ & $1.834(3)$ \\
\hline$P(3)-C(52)$ & $1.827(3)$ \\
\hline $\mathrm{P}(3)-\mathrm{C}(40)$ & $1.830(3)$ \\
\hline $\mathrm{P}(3)-\mathrm{C}(46)$ & $1.832(3)$ \\
\hline $\mathrm{F}(1)-\mathrm{C}(1)$ & $1.353(3)$ \\
\hline $\mathrm{F}(2)-\mathrm{C}(1)$ & $1.352(3)$ \\
\hline $\mathrm{F}(3)-\mathrm{C}(2)$ & $1.346(3)$ \\
\hline $\mathrm{F}(4)-\mathrm{C}(2)$ & $1.349(3)$ \\
\hline $\mathrm{F}(5)-\mathrm{C}(3)$ & $1.347(3)$ \\
\hline $\mathrm{F}(6)-\mathrm{C}(3)$ & $1.345(3)$ \\
\hline$C(4)-C(5)$ & $1.392(4)$ \\
\hline $\mathrm{C}(4)-\mathrm{C}(9)$ & $1.397(4)$ \\
\hline$C(5)-C(6)$ & $1.384(4)$ \\
\hline$C(6)-C(7)$ & $1.385(5)$ \\
\hline $\mathrm{C}(7)-\mathrm{C}(8)$ & $1.382(5)$ \\
\hline $\mathrm{C}(8)-\mathrm{C}(9)$ & $1.390(4)$ \\
\hline $\mathrm{C}(10)-\mathrm{C}(11)$ & $1.395(4)$ \\
\hline $\mathrm{C}(10)-\mathrm{C}(15)$ & $1.398(4)$ \\
\hline
\end{tabular}




$$
\begin{aligned}
& \mathrm{C}(11)-\mathrm{C}(12) \quad 1.395(4) \\
& \text { C(12)-C(13) 1.386(5) } \\
& \text { C(13)-C(14) 1.380(5) } \\
& \text { C(14)-C(15) 1.384(4) } \\
& \text { C(16)-C(21) 1.395(4) } \\
& \text { C(16)-C(17) 1.401(4) } \\
& \text { C(17)-C(18) 1.399(4) } \\
& \text { C(18)-C(19) 1.371(5) } \\
& \mathrm{C}(19)-\mathrm{C}(20) \quad 1.374(5) \\
& \mathrm{C}(20)-\mathrm{C}(21) \quad 1.391(4) \\
& \text { C(22)-C(27) 1.390(4) } \\
& \text { C(22)-C(23) 1.392(4) } \\
& \text { C(23)-C(24) 1.389(4) } \\
& \mathrm{C}(24)-\mathrm{C}(25) \quad 1.375(5) \\
& \mathrm{C}(25)-\mathrm{C}(26) \quad 1.369(5) \\
& \mathrm{C}(26)-\mathrm{C}(27) \quad 1.394(4) \\
& \text { C(28)-C(33) 1.392(4) } \\
& \text { C(28)-C(29) 1.396(4) } \\
& \text { C(29)-C(30) 1.388(4) } \\
& \mathrm{C}(30)-\mathrm{C}(31) \quad 1.377(4) \\
& \text { C(31)-C(32) 1.383(5) } \\
& \mathrm{C}(32)-\mathrm{C}(33) \quad 1.394(4) \\
& \text { C(34)-C(35) 1.391(4) } \\
& \text { C(34)-C(39) 1.395(4) } \\
& \text { C(35)-C(36) 1.406(4) } \\
& \text { C(36)-C(37) 1.380(5) } \\
& \text { C(37)-C(38) 1.377(5) } \\
& \text { C(38)-C(39) 1.388(4) } \\
& \text { C(40)-C(45) 1.391(4) } \\
& \text { C(40)-C(41) 1.405(4) }
\end{aligned}
$$




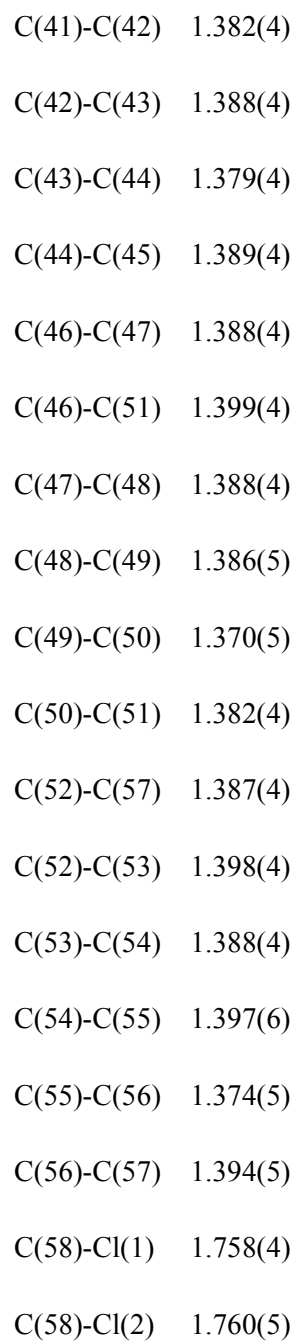

\section{Bond angles $\left[^{\circ}\right]$ for Complex A:}

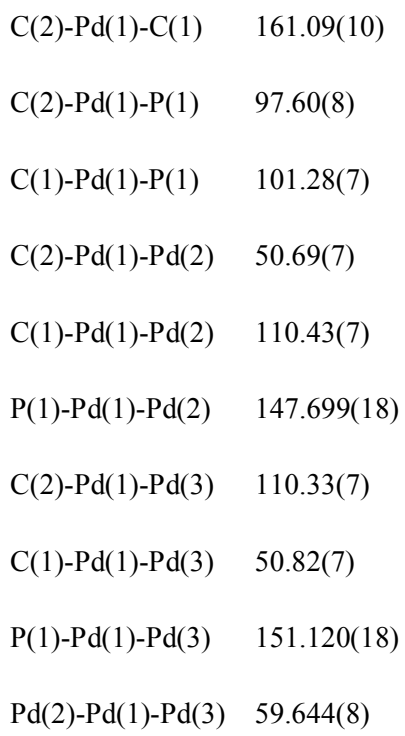




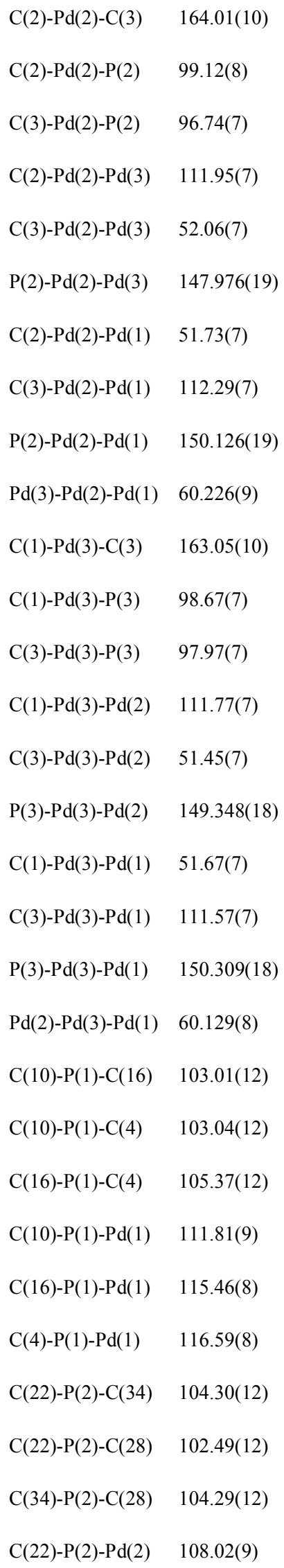




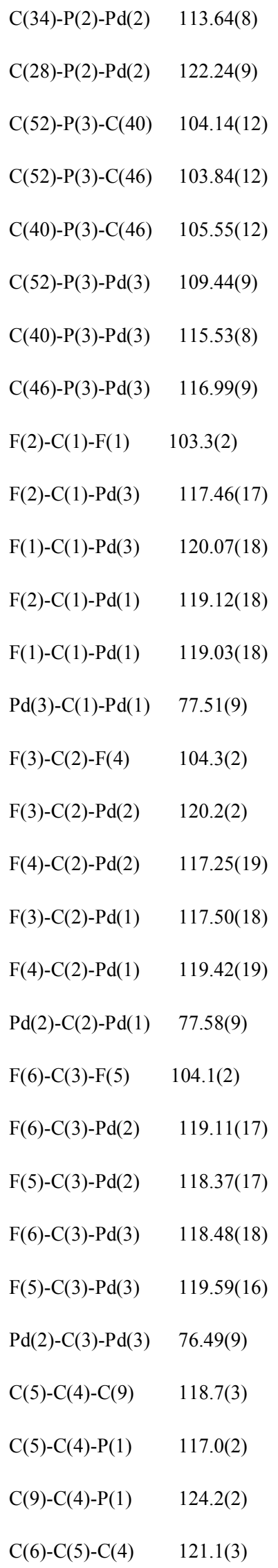




$$
\begin{aligned}
& \mathrm{C}(5)-\mathrm{C}(6)-\mathrm{C}(7) \quad 119.8(3) \\
& \text { C(8)-C(7)-C(6) 119.8(3) } \\
& \text { C(7)-C(8)-C(9) 120.6(3) } \\
& \text { C(8)-C(9)-C(4) 120.0(3) } \\
& \text { C(11)-C(10)-C(15) 119.3(3) } \\
& \text { C(11)-C(10)-P(1) 118.8(2) } \\
& \text { C(15)-C(10)-P(1) 121.9(2) } \\
& \mathrm{C}(10)-\mathrm{C}(11)-\mathrm{C}(12) \quad 120.0(3) \\
& \text { C(13)-C(12)-C(11) 120.0(3) } \\
& \text { C(14)-C(13)-C(12) 120.1(3) } \\
& \text { C(13)-C(14)-C(15) 120.5(3) } \\
& \text { C(14)-C(15)-C(10) 120.1(3) } \\
& \text { C(21)-C(16)-C(17) 118.7(2) } \\
& \mathrm{C}(21)-\mathrm{C}(16)-\mathrm{P}(1) \quad 121.8(2) \\
& \text { C(17)-C(16)-P(1) 119.3(2) } \\
& \text { C(18)-C(17)-C(16) 119.6(3) } \\
& \text { C(19)-C(18)-C(17) 120.8(3) } \\
& \text { C(18)-C(19)-C(20) 120.0(3) } \\
& \text { C(19)-C(20)-C(21) 120.3(3) } \\
& \mathrm{C}(20)-\mathrm{C}(21)-\mathrm{C}(16) \quad 120.5(3) \\
& \text { C(27)-C(22)-C(23) 118.9(3) } \\
& \mathrm{C}(27)-\mathrm{C}(22)-\mathrm{P}(2) \quad 123.0(2) \\
& \text { C(23)-C(22)-P(2) 118.1(2) } \\
& \text { C(24)-C(23)-C(22) 120.3(3) } \\
& \text { C(25)-C(24)-C(23) 120.3(3) } \\
& \mathrm{C}(26)-\mathrm{C}(25)-\mathrm{C}(24) \quad 120.0(3) \\
& \mathrm{C}(25)-\mathrm{C}(26)-\mathrm{C}(27) \quad 120.5(3) \\
& \text { C(22)-C(27)-C(26) 120.0(3) } \\
& \text { C(33)-C(28)-C(29) 118.2(2) } \\
& \mathrm{C}(33)-\mathrm{C}(28)-\mathrm{P}(2) \quad 124.0(2)
\end{aligned}
$$




$$
\begin{aligned}
& \text { C(29)-C(28)-P(2) 117.7(2) } \\
& \text { C(30)-C(29)-C(28) 120.8(3) } \\
& \text { C(31)-C(30)-C(29) 120.5(3) } \\
& \text { C(30)-C(31)-C(32) 119.6(3) } \\
& \text { C(31)-C(32)-C(33) 120.1(3) } \\
& \text { C(28)-C(33)-C(32) 120.8(3) } \\
& \text { C(35)-C(34)-C(39) 119.9(2) } \\
& \mathrm{C}(35)-\mathrm{C}(34)-\mathrm{P}(2) \quad 121.8(2) \\
& \text { C(39)-C(34)-P(2) 118.3(2) } \\
& \text { C(34)-C(35)-C(36) 119.3(3) } \\
& \text { C(37)-C(36)-C(35) 120.0(3) } \\
& \text { C(38)-C(37)-C(36) 120.6(3) } \\
& \text { C(37)-C(38)-C(39) 120.0(3) } \\
& \mathrm{C}(38)-\mathrm{C}(39)-\mathrm{C}(34) \quad 120.1(3) \\
& \text { C(45)-C(40)-C(41) 118.4(2) } \\
& \text { C(45)-C(40)-P(3) 124.1(2) } \\
& \text { C(41)-C(40)-P(3) 117.1(2) } \\
& \text { C(42)-C(41)-C(40) 120.7(3) } \\
& \text { C(41)-C(42)-C(43) 120.2(3) } \\
& \text { C(44)-C(43)-C(42) 119.6(3) } \\
& \text { C(43)-C(44)-C(45) 120.6(3) } \\
& \text { C(44)-C(45)-C(40) 120.5(3) } \\
& \text { C(47)-C(46)-C(51) 118.4(3) } \\
& \text { C(47)-C(46)-P(3) 118.8(2) } \\
& \mathrm{C}(51)-\mathrm{C}(46)-\mathrm{P}(3) \quad 122.7(2) \\
& \text { C(48)-C(47)-C(46) 120.9(3) } \\
& \text { C(49)-C(48)-C(47) 119.5(3) } \\
& \text { C(50)-C(49)-C(48) 120.2(3) } \\
& \text { C(49)-C(50)-C(51) 120.5(3) } \\
& \mathrm{C}(50)-\mathrm{C}(51)-\mathrm{C}(46) \quad 120.4(3)
\end{aligned}
$$




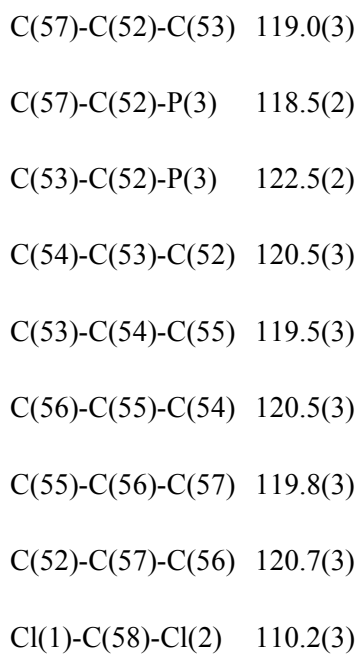

\section{The observation of $\left[\left(\mathrm{Ph}_{3} \mathrm{P}\right)_{2} \mathrm{PdCF} \mathrm{F}_{2} \mathrm{H}\right]^{+}$complex}

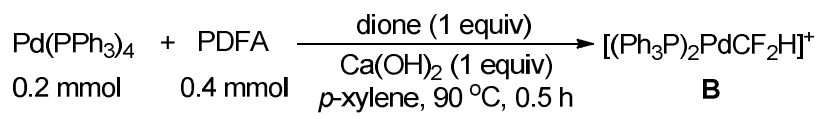

A dried Schlenk tube was charged $\mathrm{Ph}_{3} \mathrm{P}^{+} \mathrm{CF}_{2} \mathrm{CO}_{2}{ }^{-}(143 \mathrm{mg}, 0.4 \mathrm{mmol})$, 1,3-Cyclopendione (19.6 mg, $0.2 \mathrm{mmol}), \mathrm{Ca}(\mathrm{OH})_{2}(14.8 \mathrm{mg}, 0.2 \mathrm{mmol}), \mathrm{Pd}\left(\mathrm{PPh}_{3}\right)_{4}$ (231 mg, $0.2 \mathrm{mmol})$ and $p$-xylene $(2 \mathrm{~mL})$. The resulting mixture was stirred at $90{ }^{\circ} \mathrm{C}$ for $0.5 \mathrm{~h}$ under $\mathrm{N}_{2}$ atmosphere. The reaction mixture was cooled to room temperature, and species B was detected by ${ }^{19} \mathrm{~F}$ NMR and HRMS (ESI). ${ }^{19} \mathrm{~F}$ NMR (376 MHz) $\delta$ $-96.04(\mathrm{~d}, J=49.5 \mathrm{~Hz})$. HRMS (ESI) calcd. for $\mathrm{C}_{37} \mathrm{H}_{31} \mathrm{~F}_{2} \mathrm{P}_{2}{ }^{102} \mathrm{Pd}[\mathrm{M}]^{+}: 677.0920$. Found: 677.0942. 
${ }^{19} \mathrm{~F} \mathrm{NMR}^{a}$
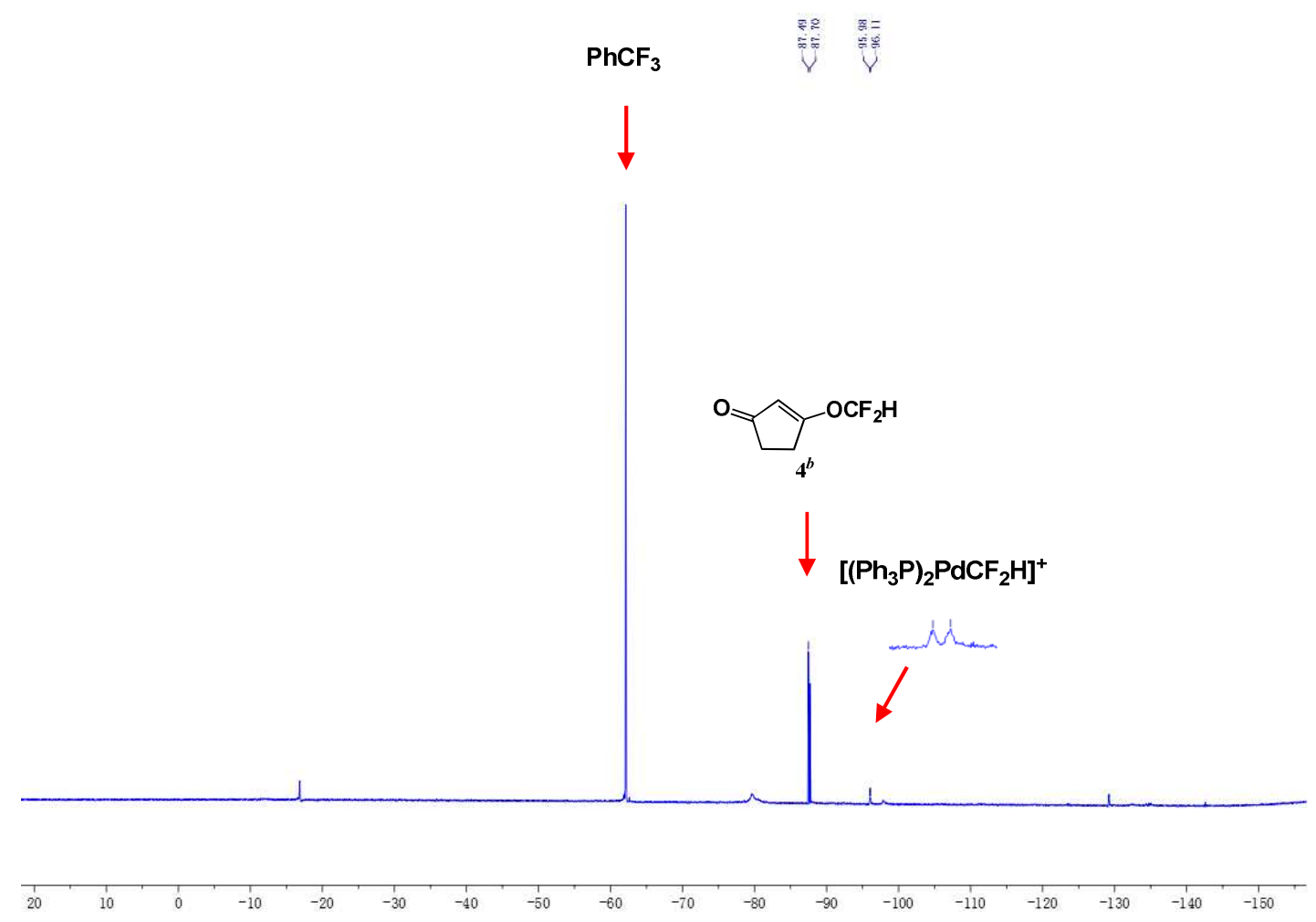

${ }^{a} \mathrm{PhCF}_{3}$ was used as an internal standard. ${ }^{b}$ Compound 4 was always produced by the reaction of 1,3-cyclopentadione with difluorocarbene.

\section{References}

(1) Brown, H. C.; Gupta, S. K. J. Am. Chem. Soc. 1975, 97, 5249.

(2) Fier, P. S.; Hartwig, J. F. J. Am. Chem. Soc. 2012, 134, 5524.

(3) Feng, Z.; Min, Q.; Zhang, X. Org. Lett. 2016, 18, 44.

(4) Su, Y.-M.; Hou, Y.; Yin, F.; Xu, Y.-M.; Li, Y.; Zheng, X.; Wang, X.-S. Org. Lett. 2014, 16, 2958.

(5) Chang, D.; Gu, Y.; Shen Q. Chem. Eur. J. 2015, $21,6074$. 
8. Copies of ${ }^{1} \mathrm{H}$ NMR, ${ }^{19}$ F NMR and ${ }^{13} \mathrm{C}$ NMR spectra ${ }^{1}$ H NMR
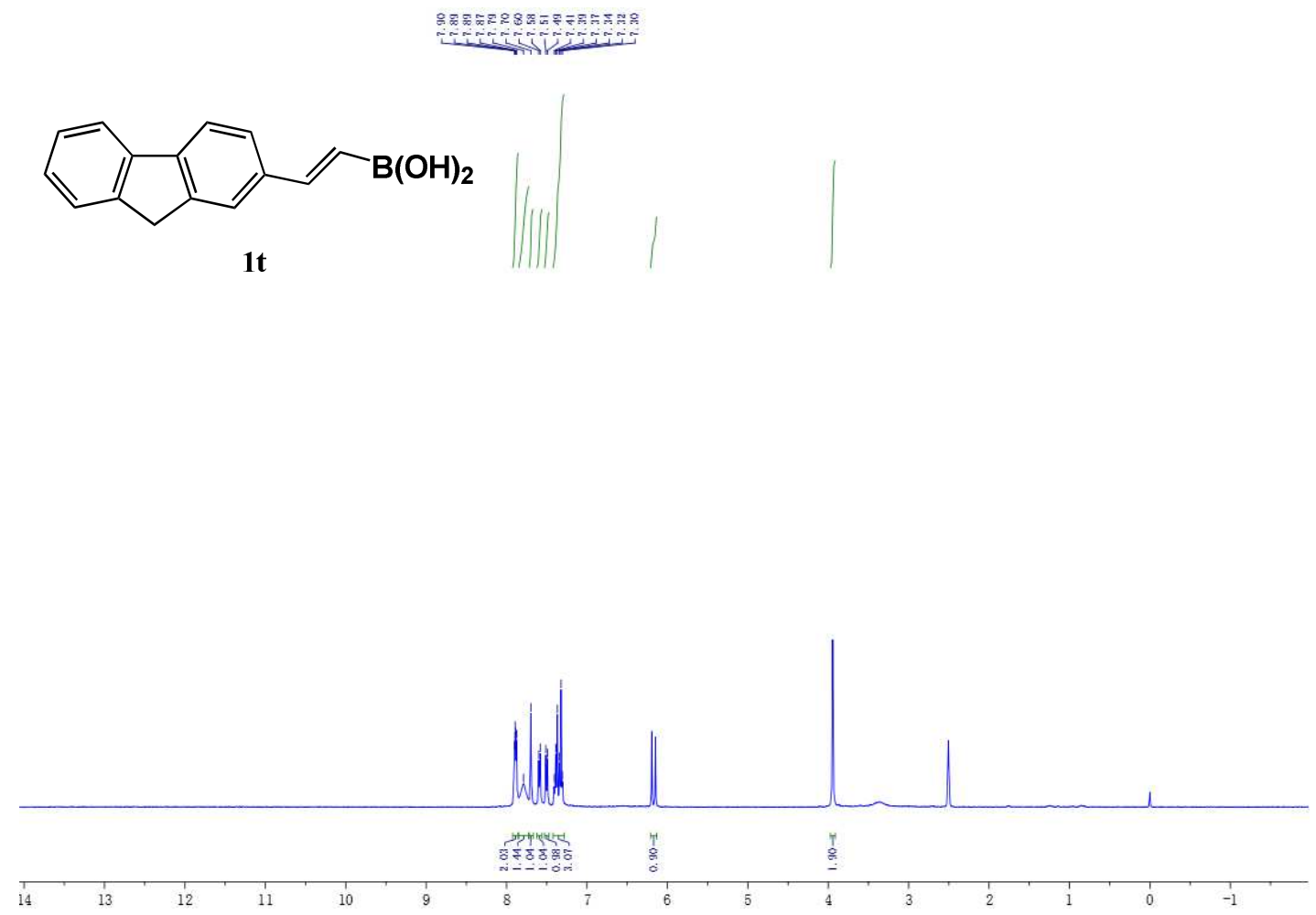

${ }^{13}$ C NMR

385

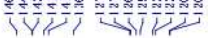
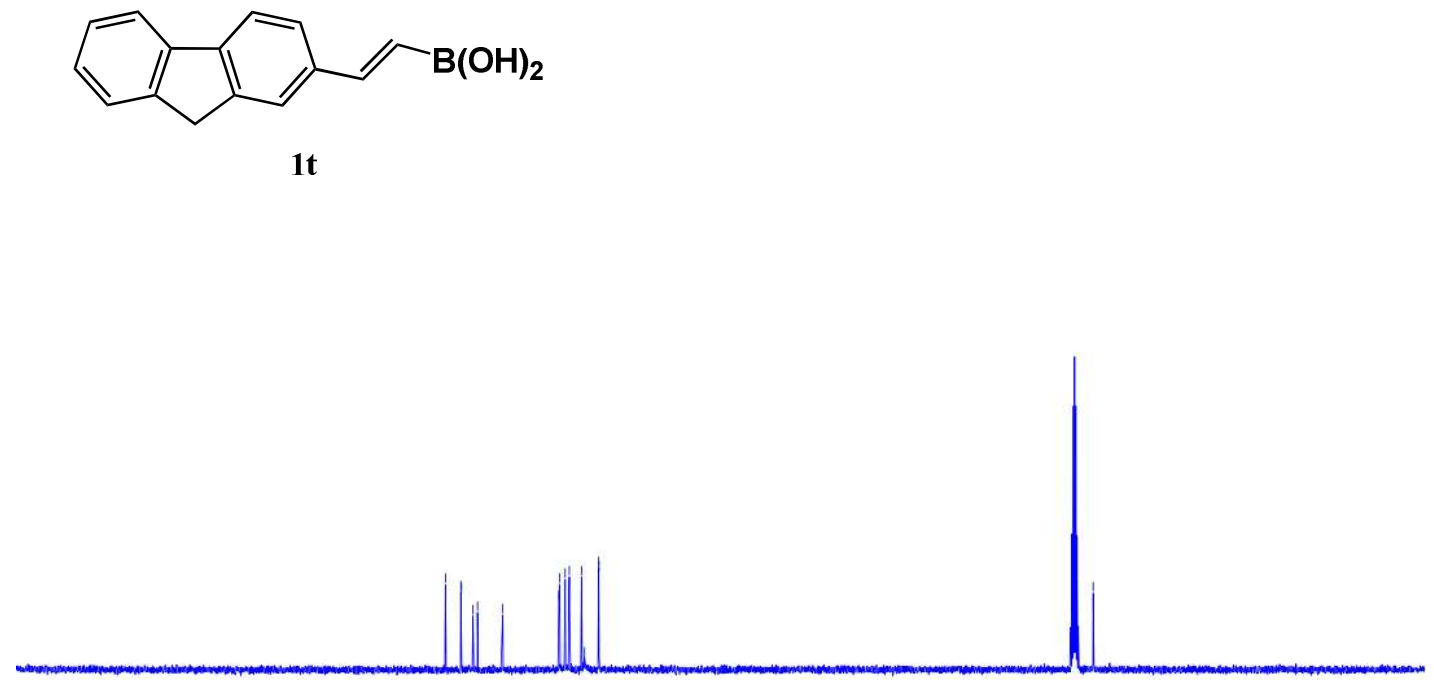

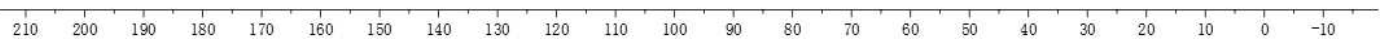




\section{${ }^{1}$ H NMR}
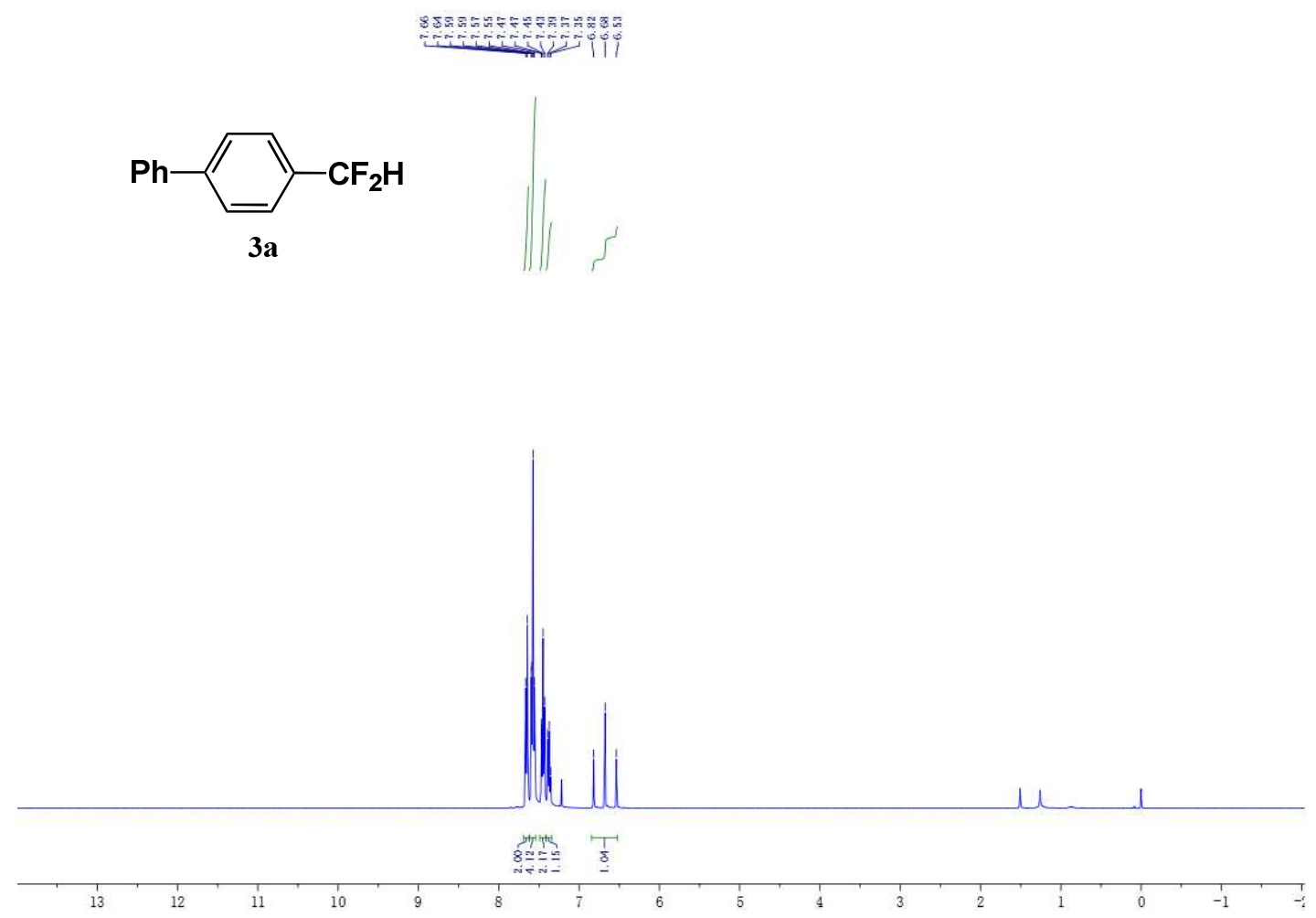

${ }^{19}$ F NMR
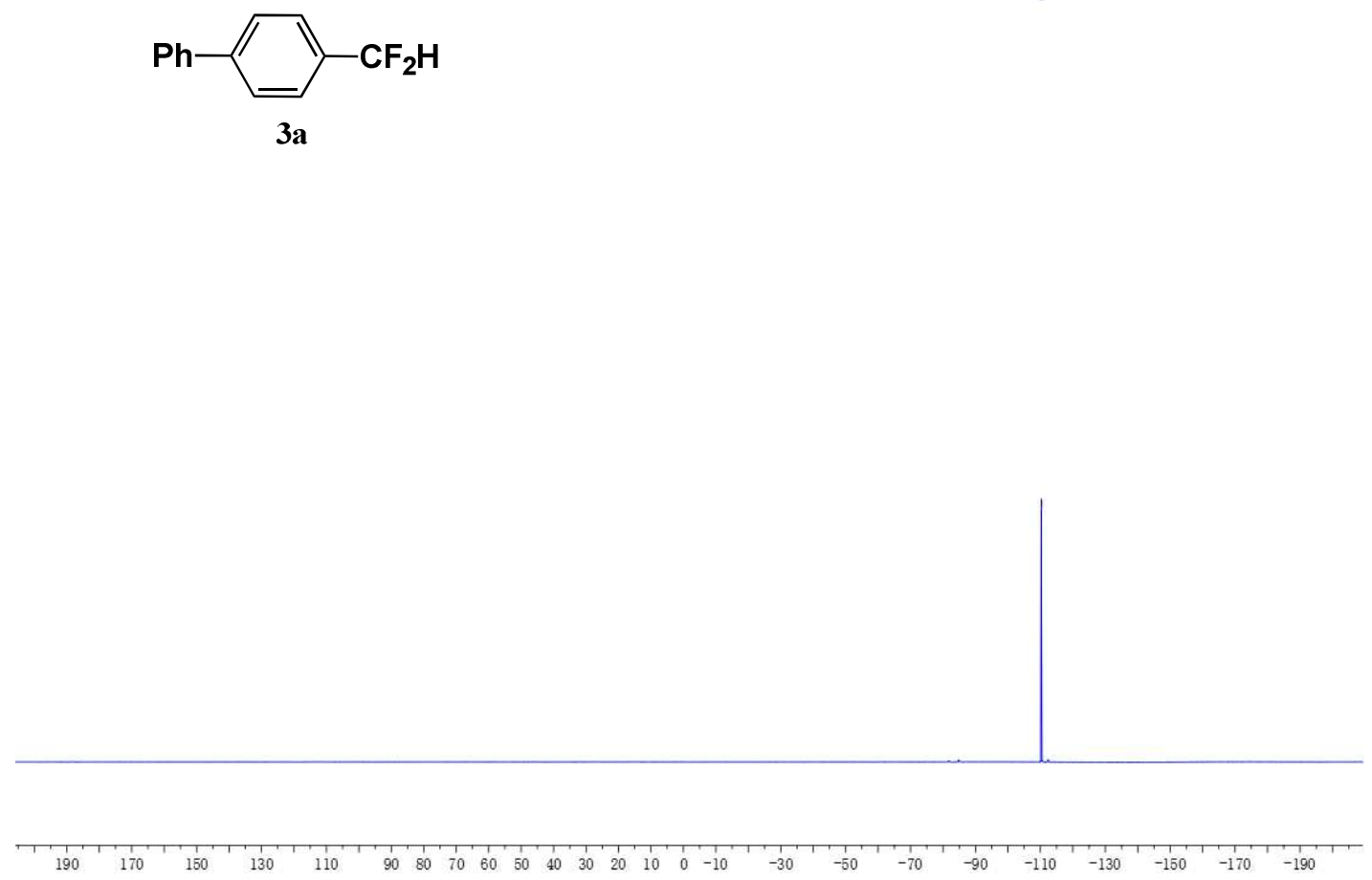


\section{${ }^{1}$ H NMR}
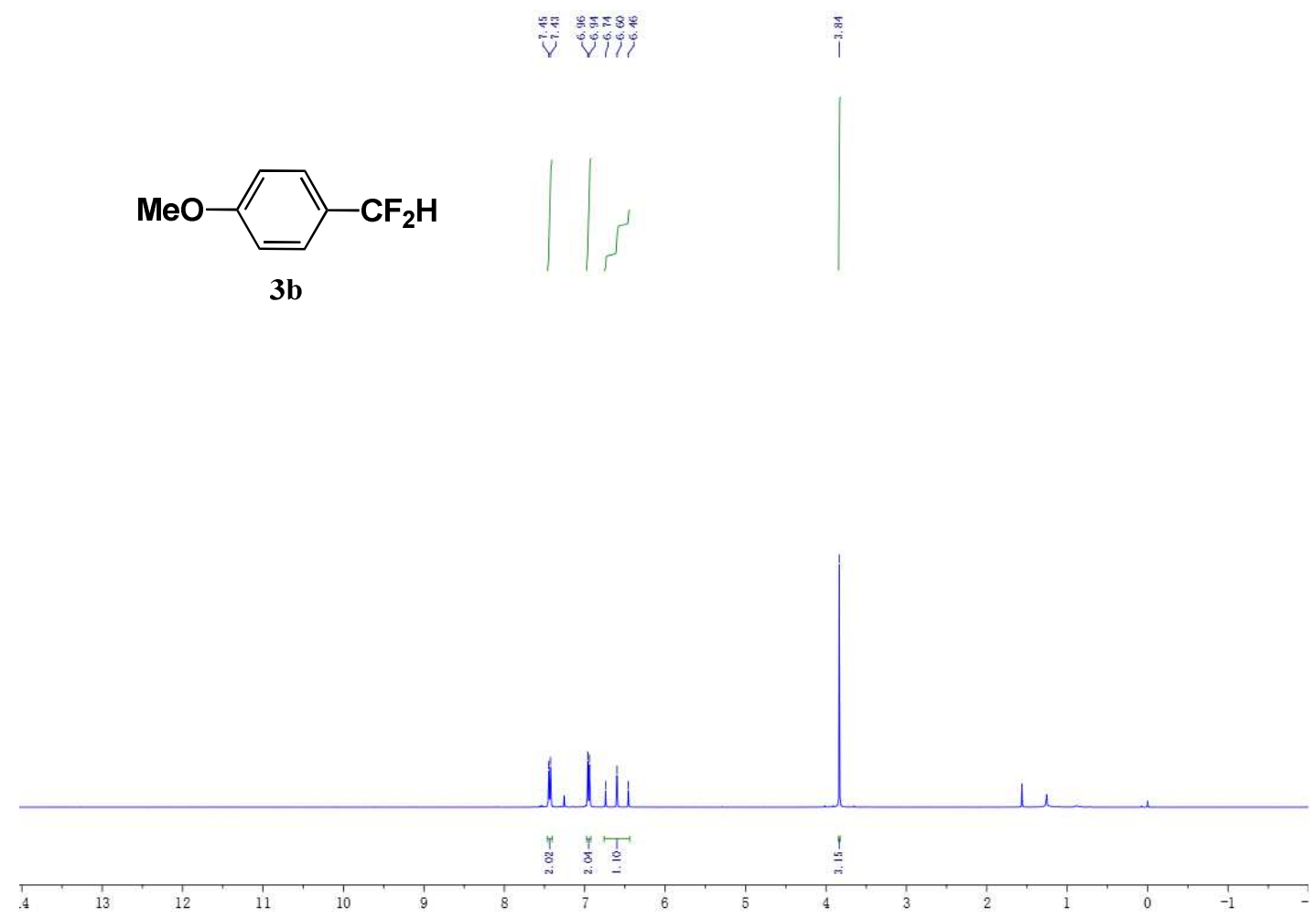

${ }^{19}$ F NMR

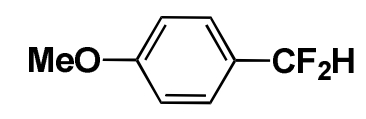

3b

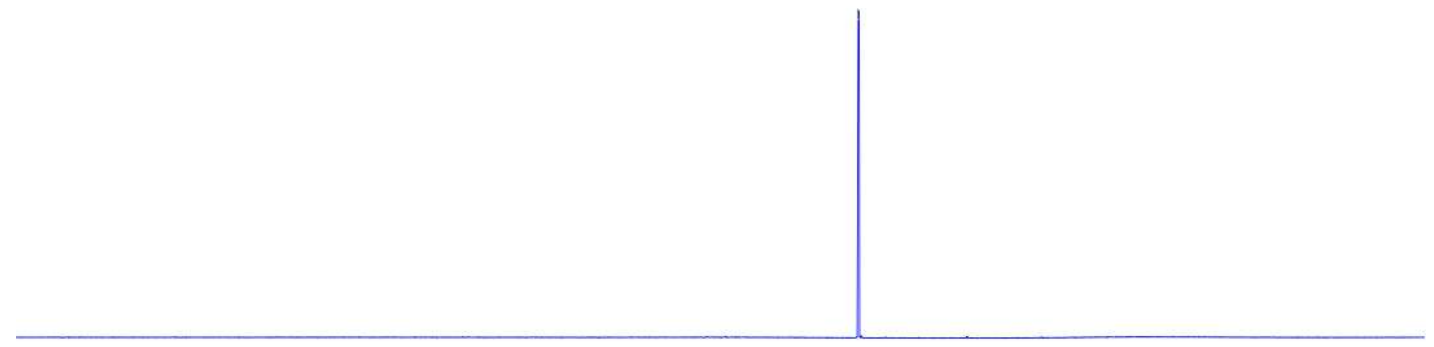

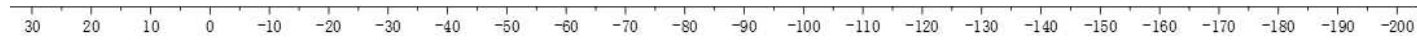


${ }^{1}$ H NMR
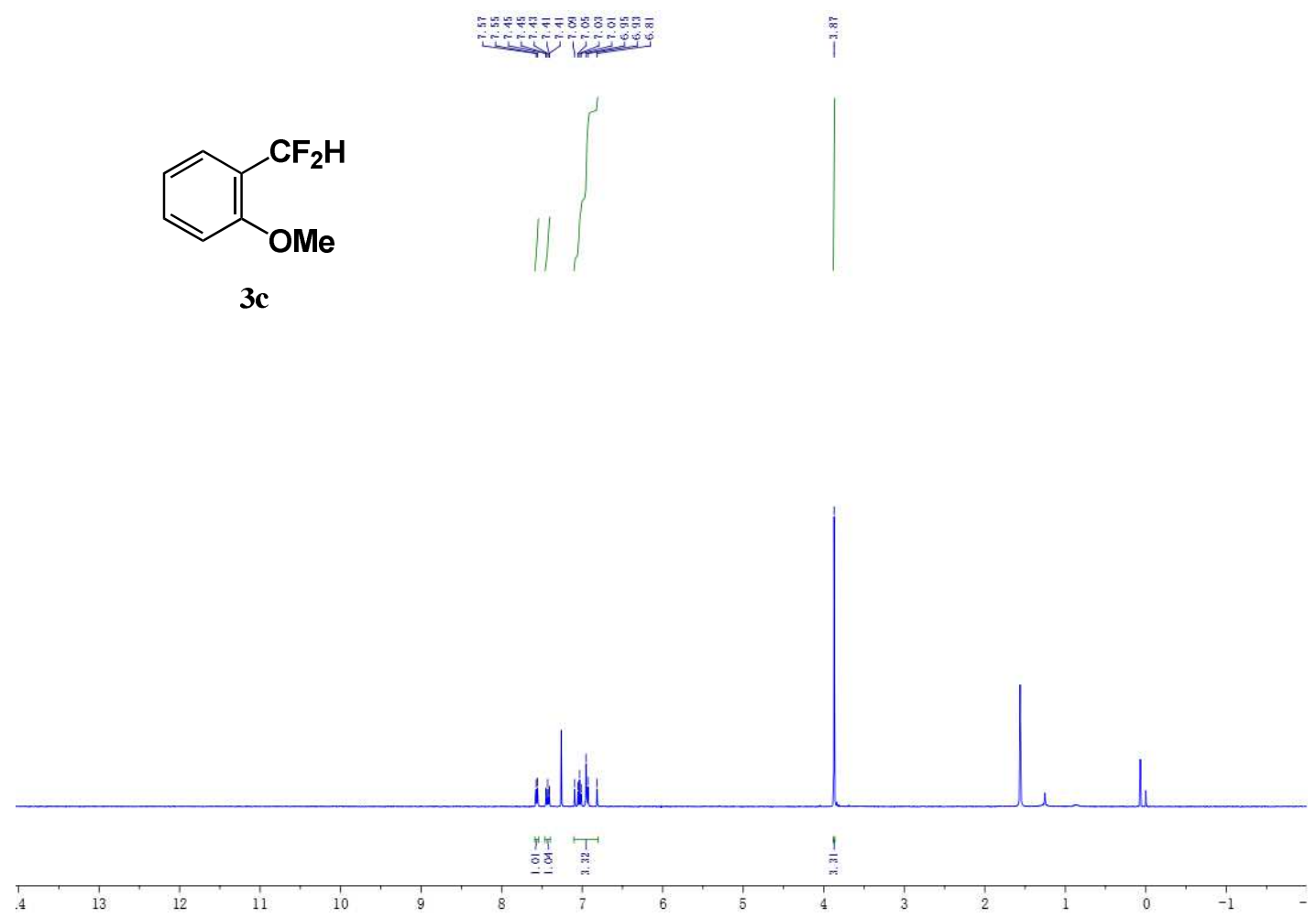

${ }^{19}$ F NMR
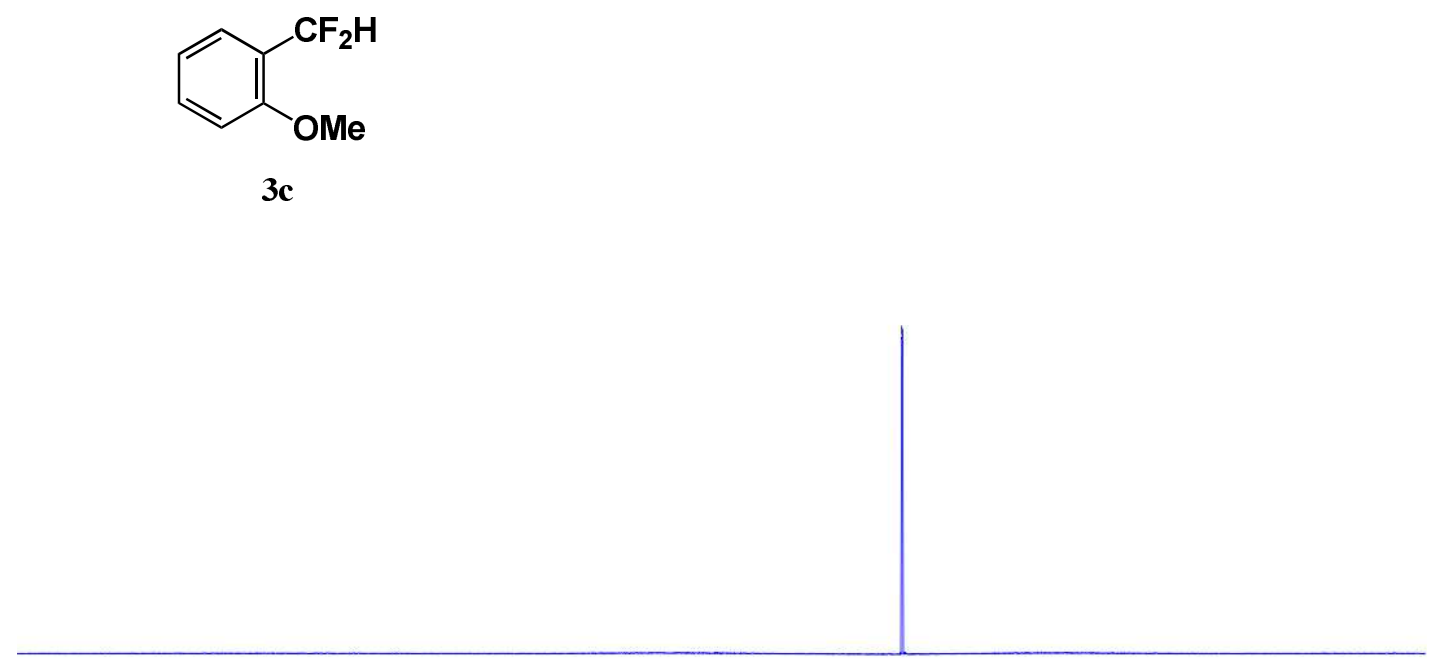

\begin{tabular}{llllllllllllllllllllllllllllll}
\hline & 1 \\
30 & 20 & 10 & 0 & -10 & -20 & -30 & -40 & -50 & -60 & -70 & -80 & -90 & -100 & -110 & -120 & -130 & -140 & -150 & -160 & -170 & -180 & -190 & -200 \\
\hline
\end{tabular} 
${ }^{1}$ H NMR
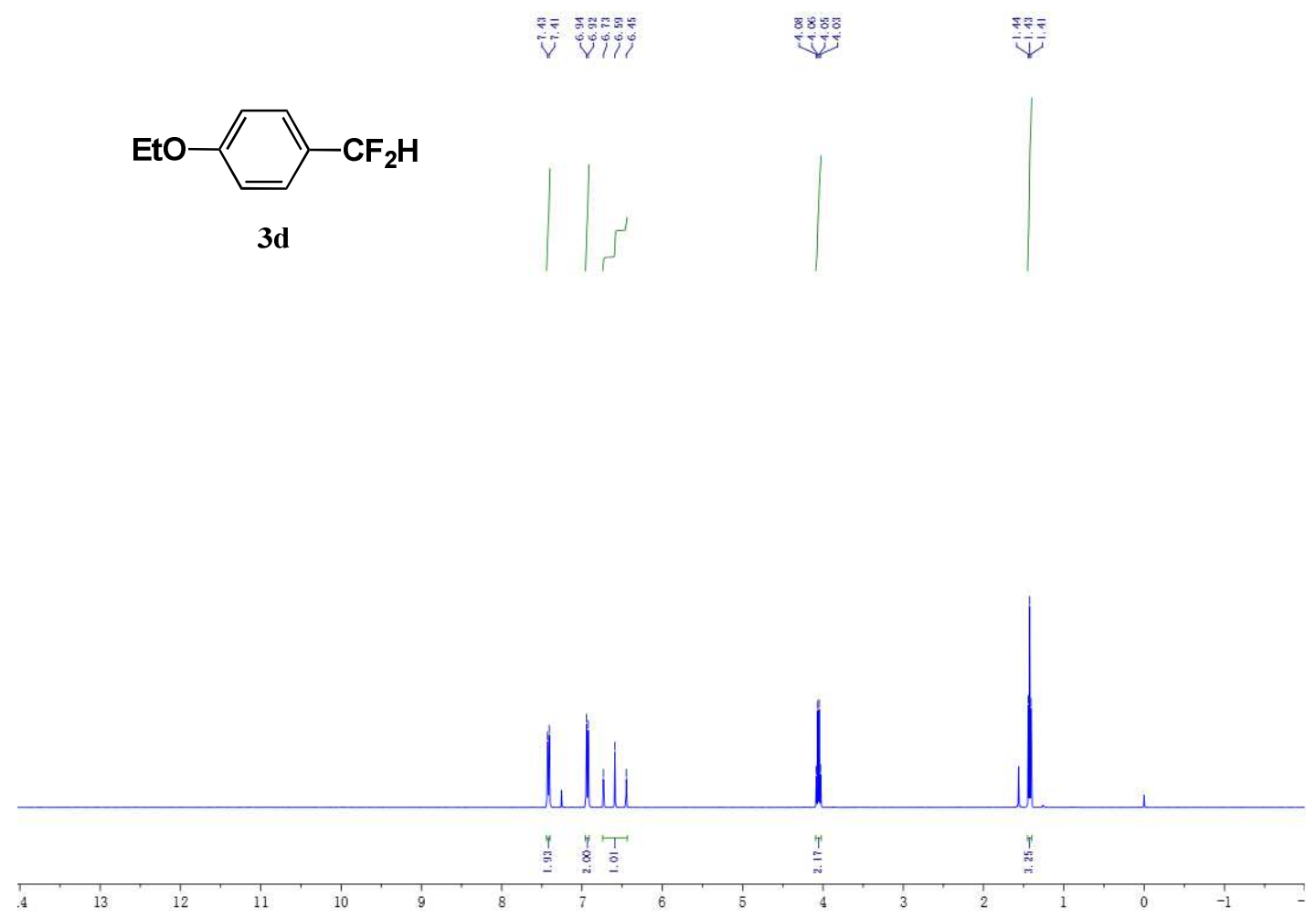

${ }^{19}$ F NMR

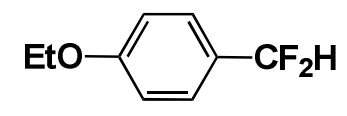

3d

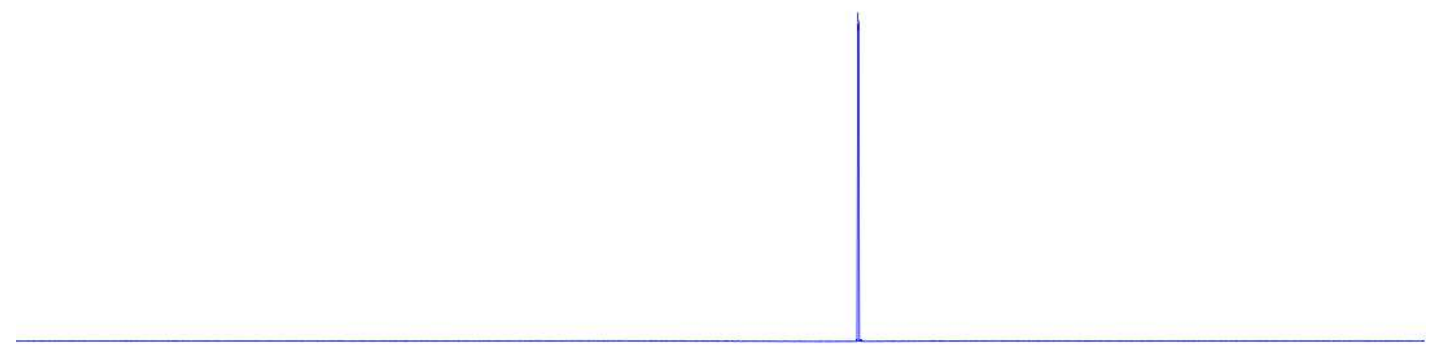

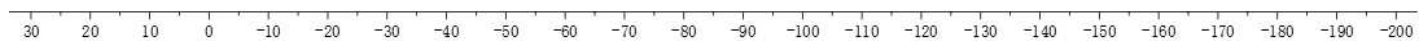




\section{${ }^{13}$ C NMR}
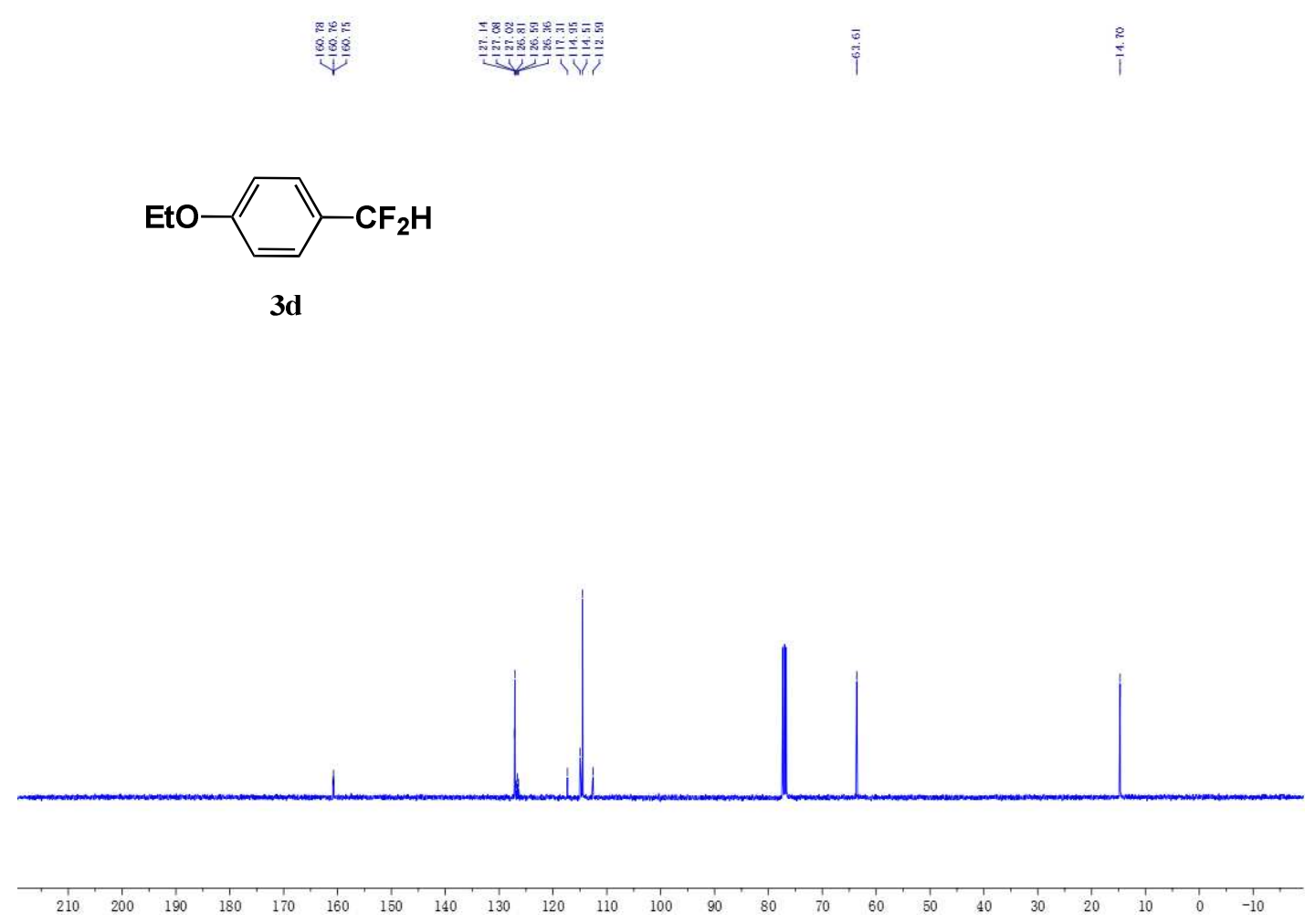

\section{${ }^{1}$ H NMR}
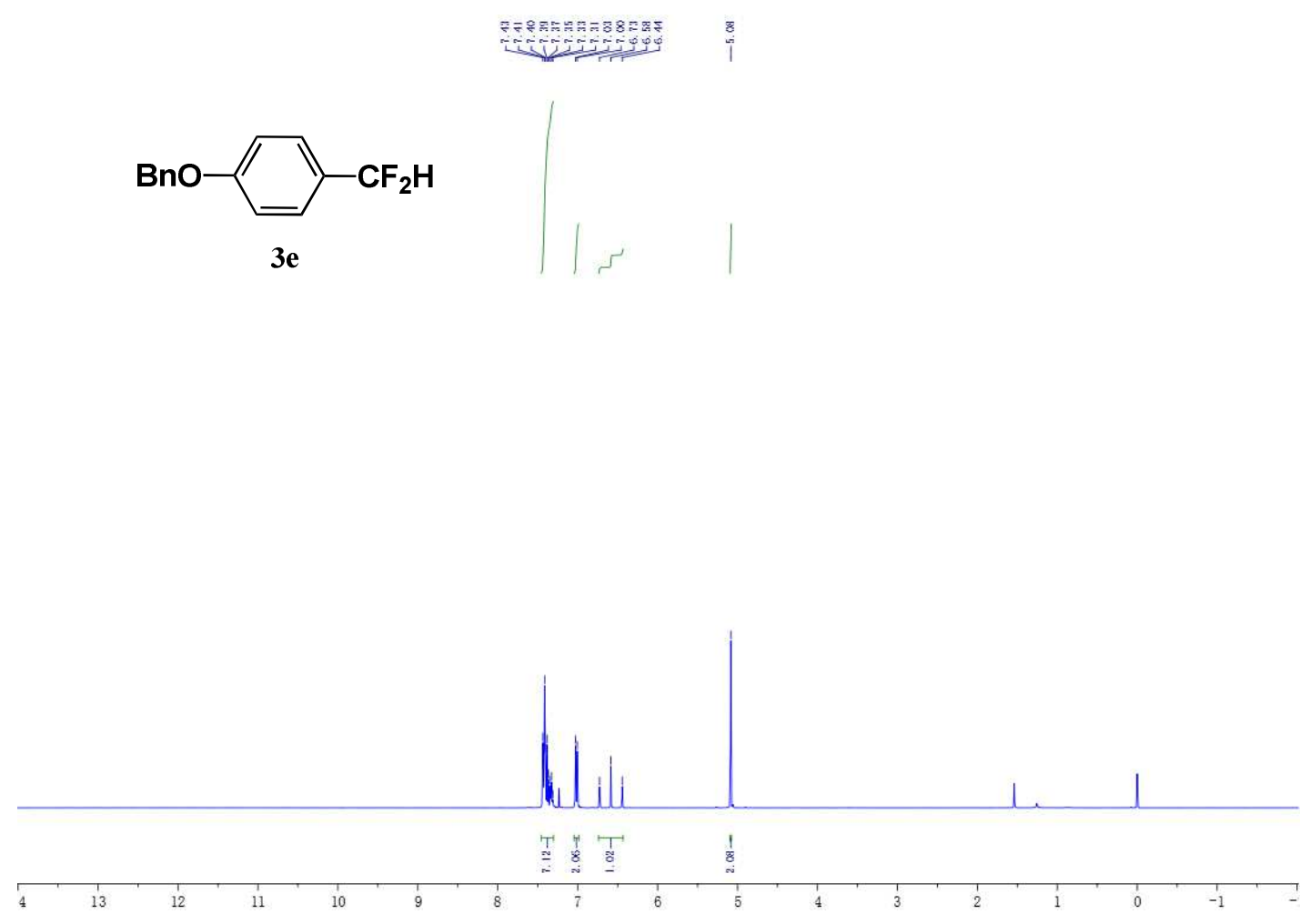


\section{${ }^{19}$ F NMR}

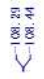
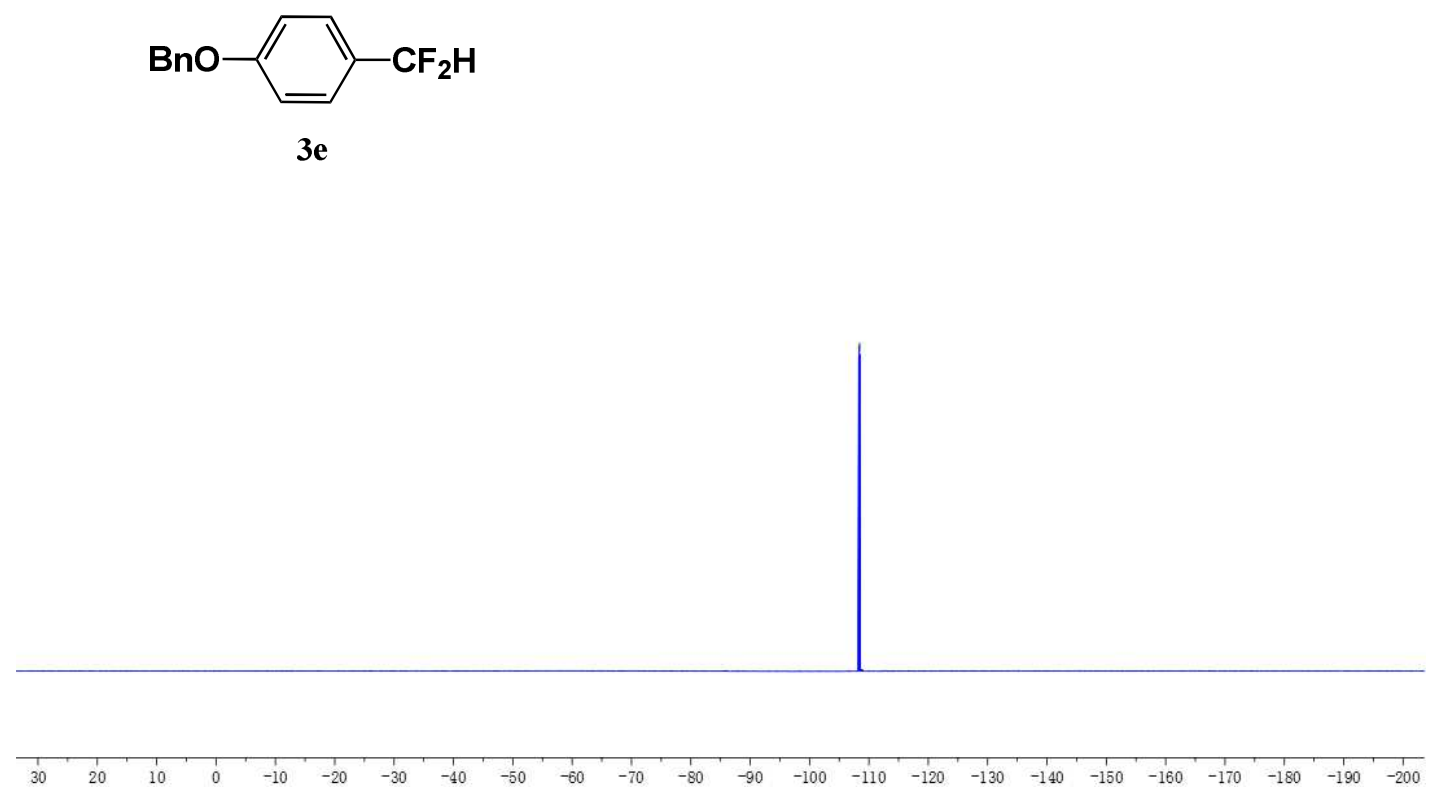

\section{${ }^{1}$ H NMR}
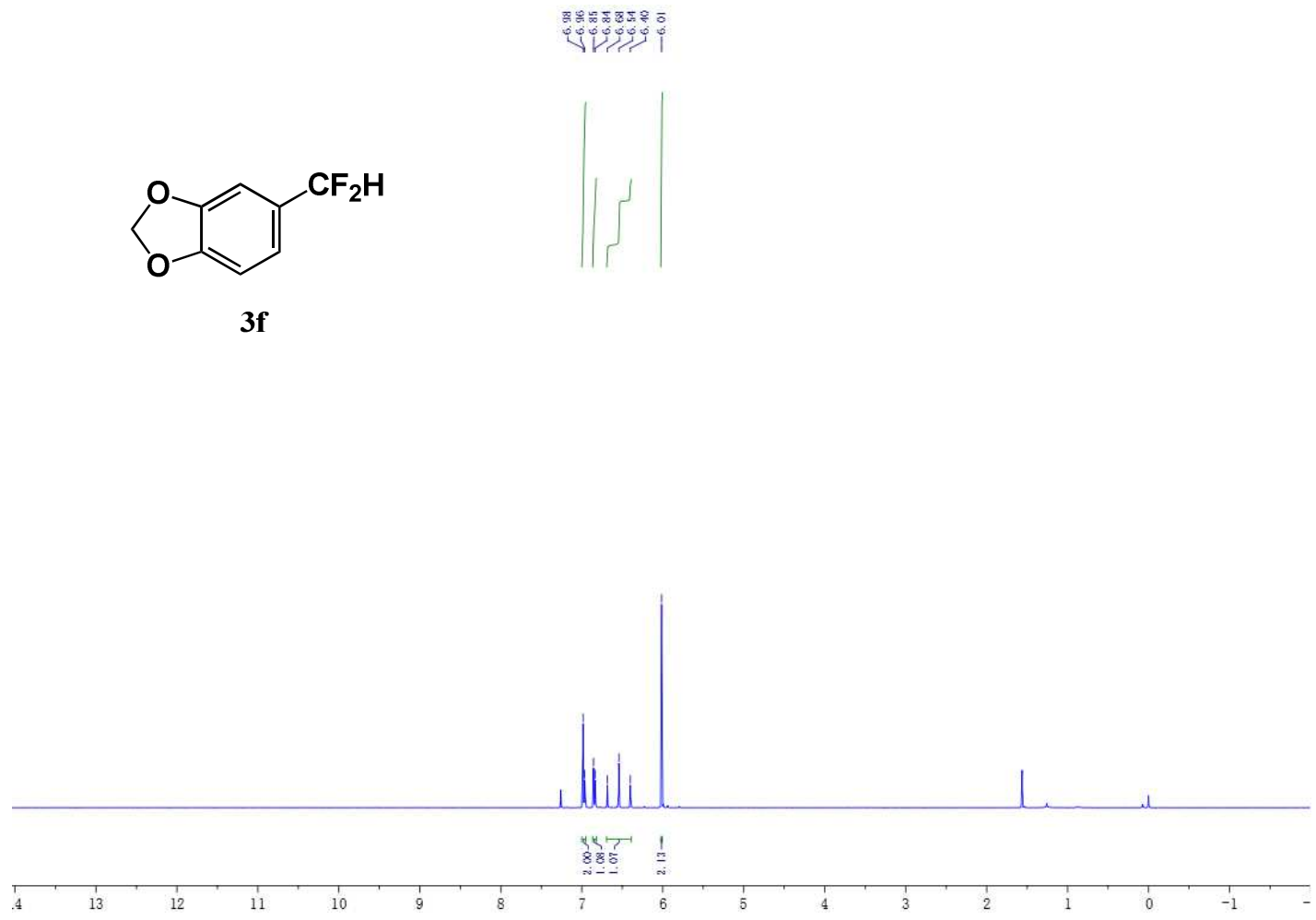


\section{${ }^{19}$ F NMR}
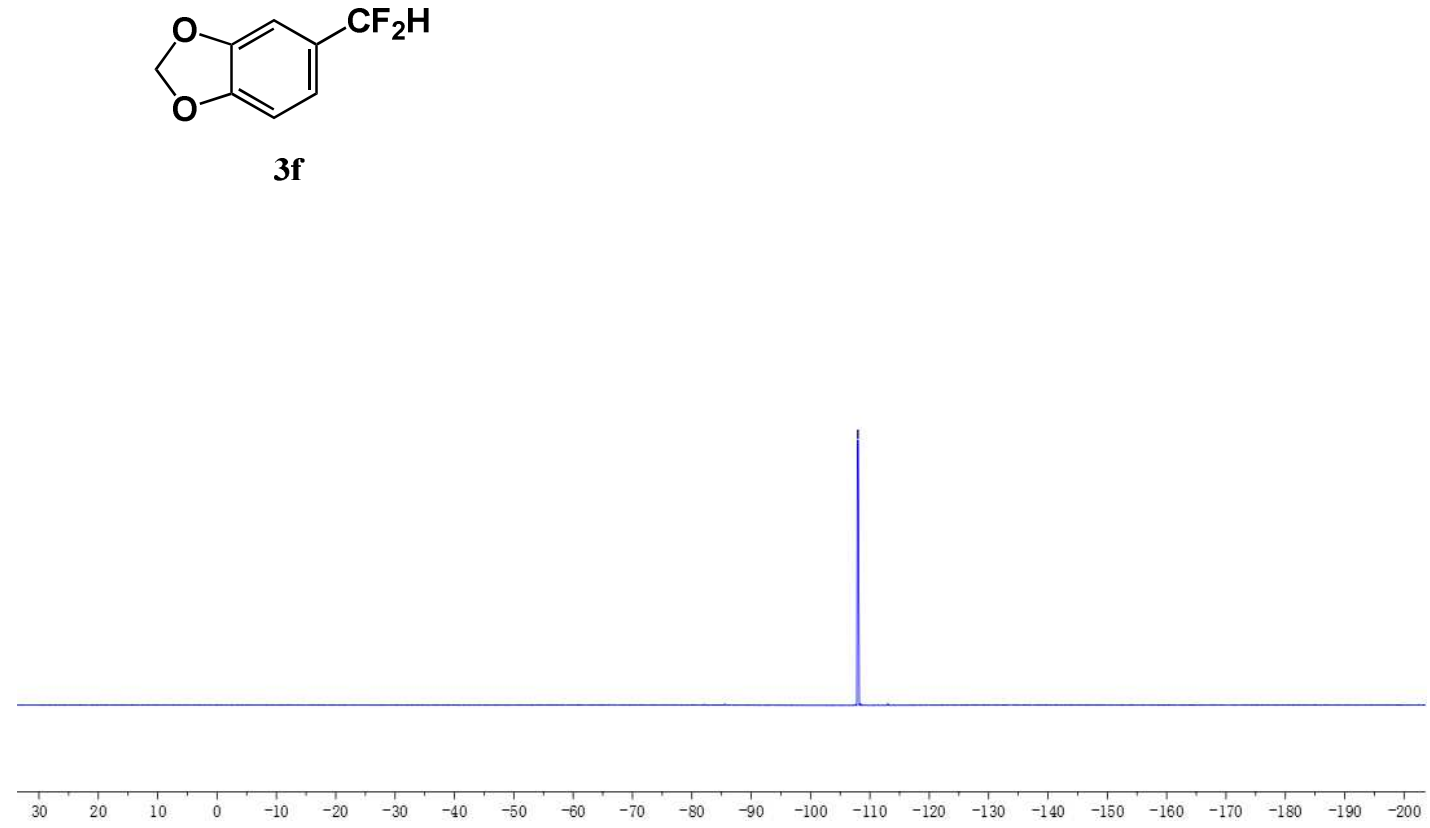

\section{${ }^{1}$ H NMR}

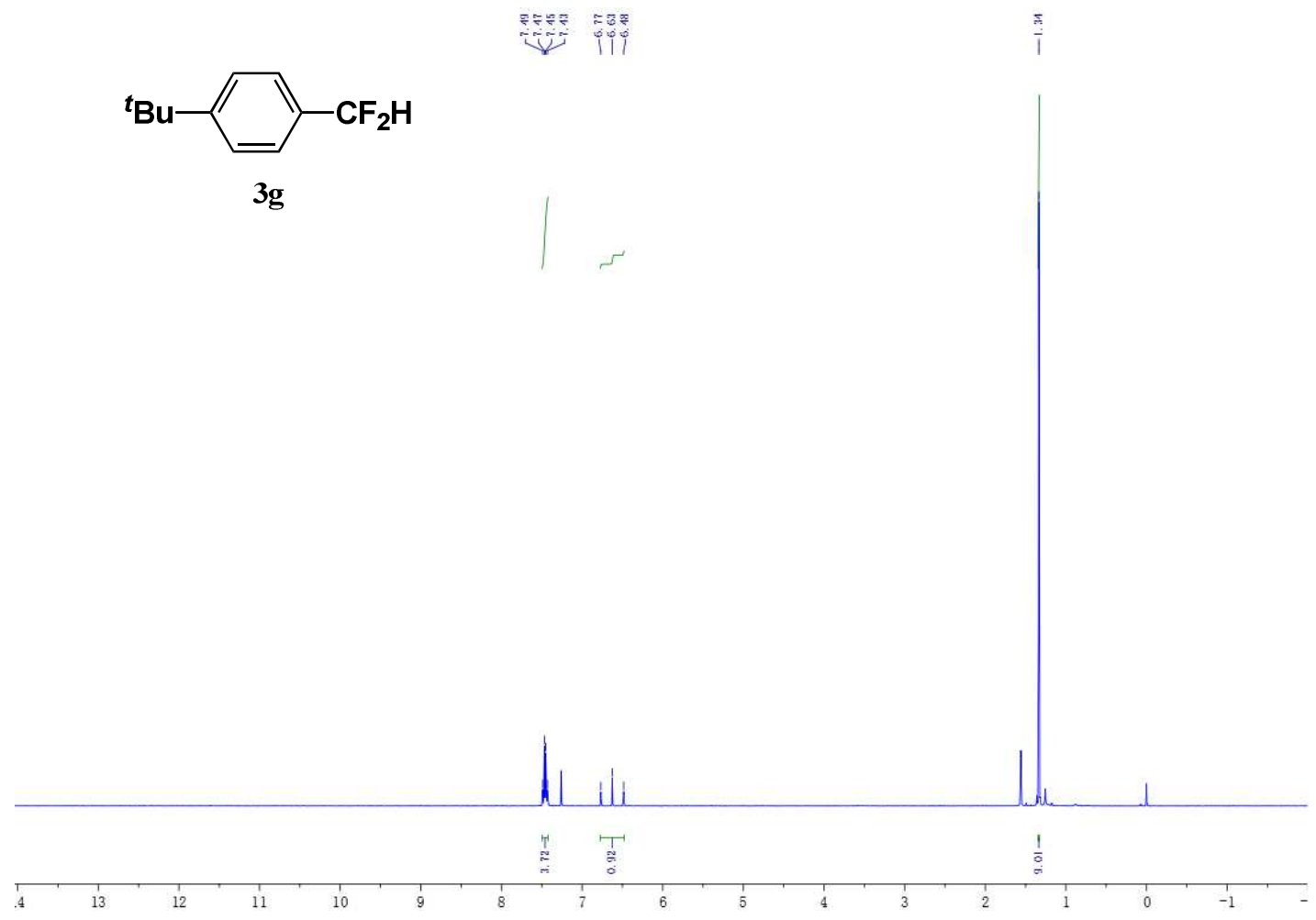




\section{${ }^{19}$ F NMR}

$\infty: 8$
$\frac{8}{8}$
$\frac{8}{7}$
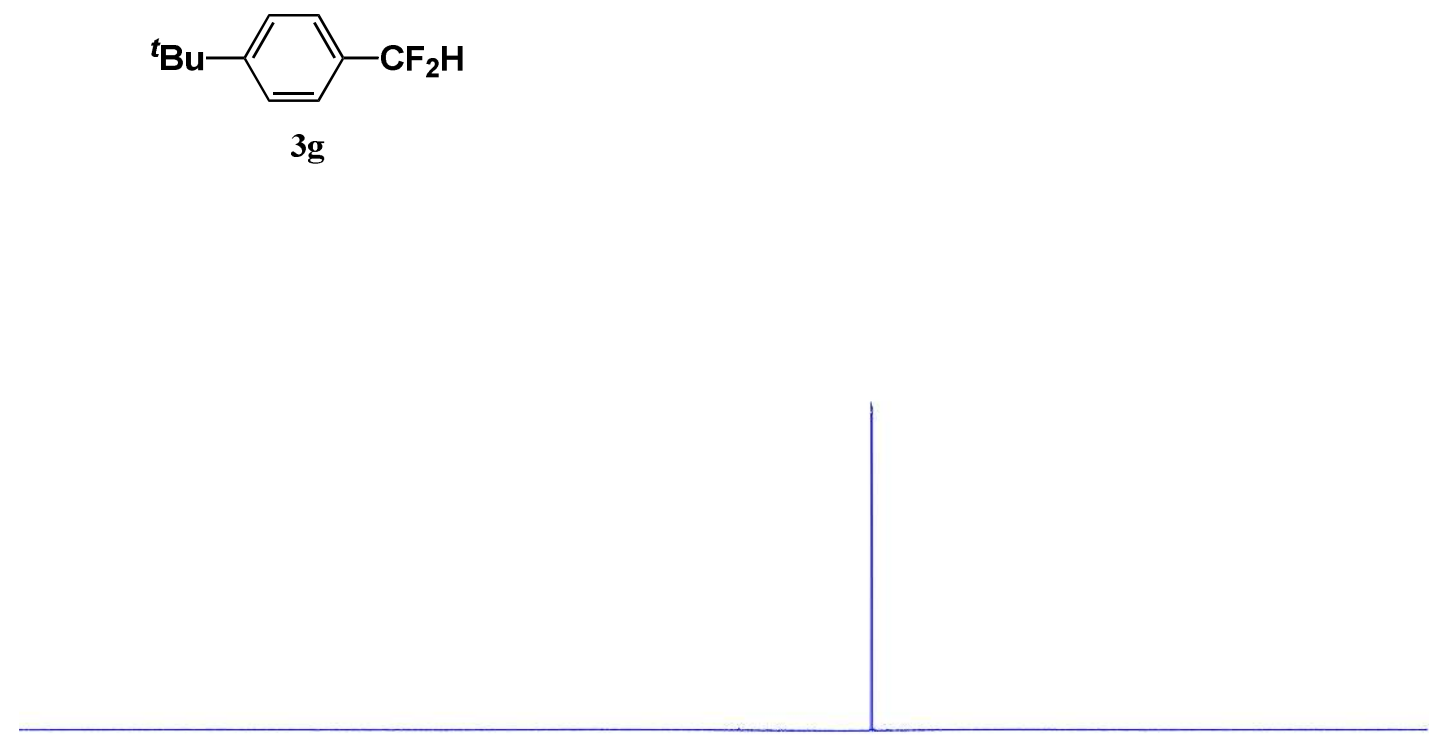

\begin{tabular}{llllllllllllllllllllllllllllll}
\hline & 1 & 10 & 10 & 0 & -10 & -20 & -30 & -40 & -50 & -60 & -70 & -80 & -90 & -100 & -110 & -120 & -130 & -140 & -150 & -160 & -170 & -180 & -190 & -200 \\
\hline
\end{tabular}

\section{${ }^{1}$ H NMR}

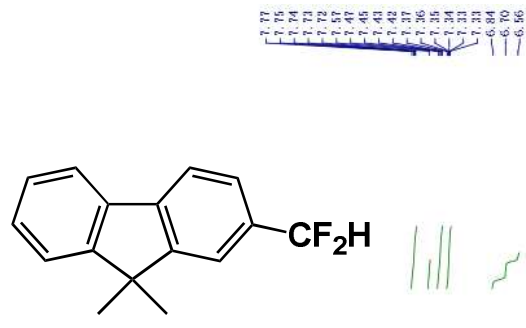

3h

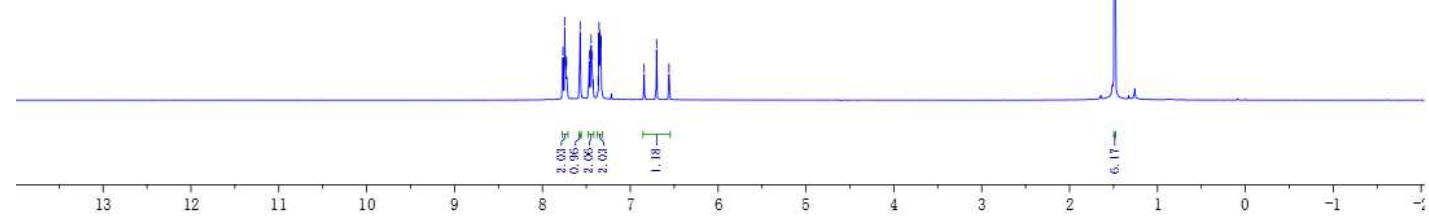




\section{${ }^{19}$ F NMR}

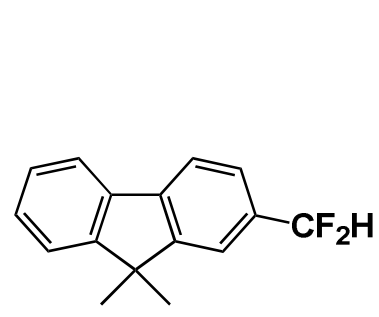

$3 h$
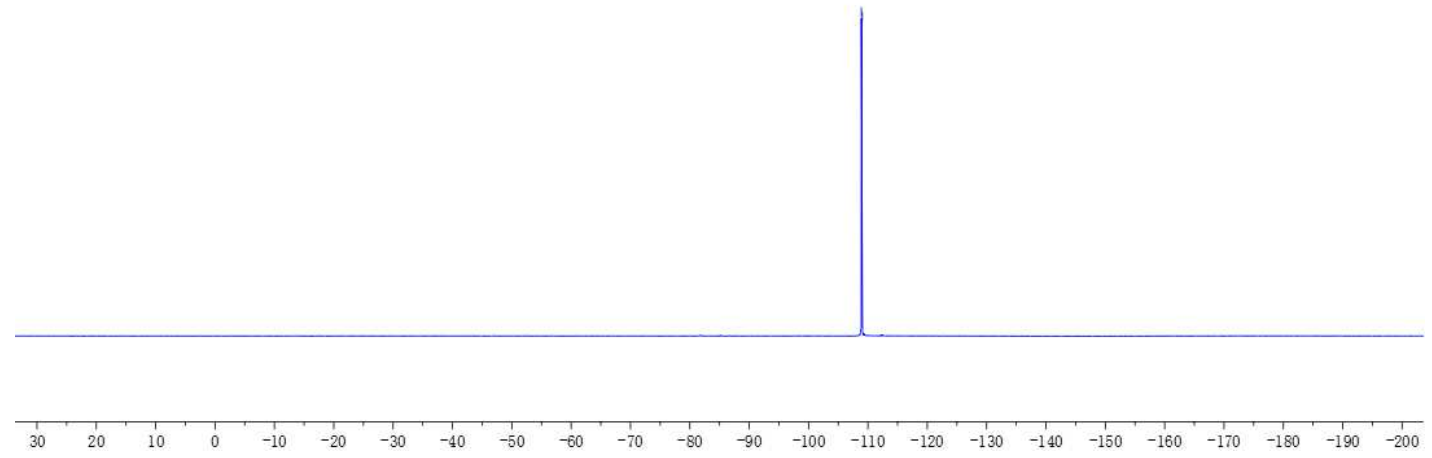

${ }^{13}$ C NMR

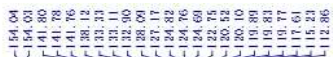

$\stackrel{8}{\stackrel{1}{i}} \stackrel{8}{i}$

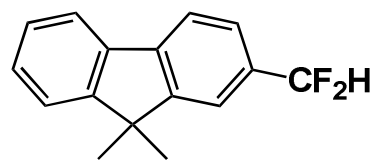

3h

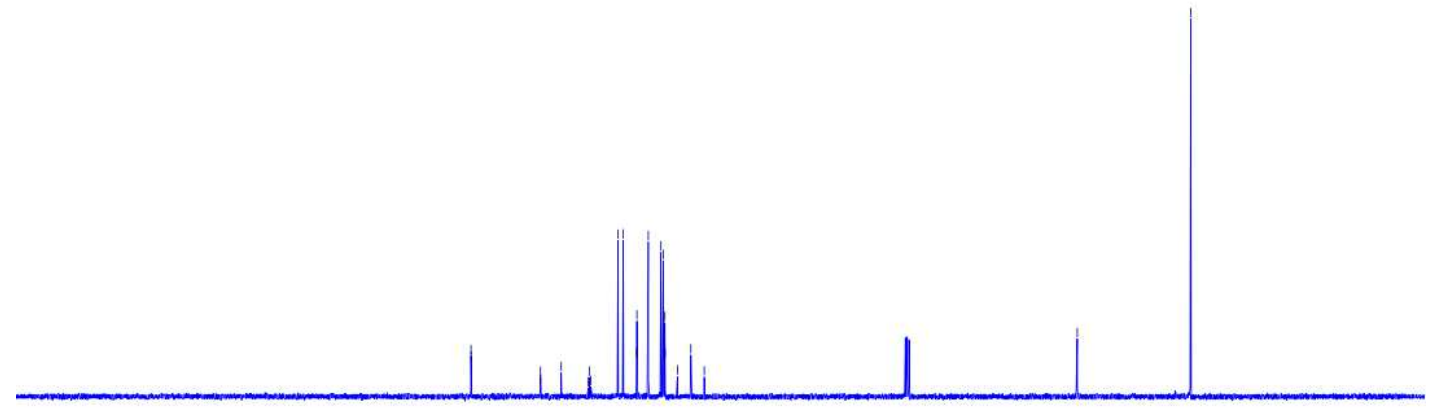

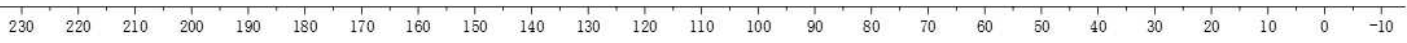




\section{${ }^{1}$ H NMR}
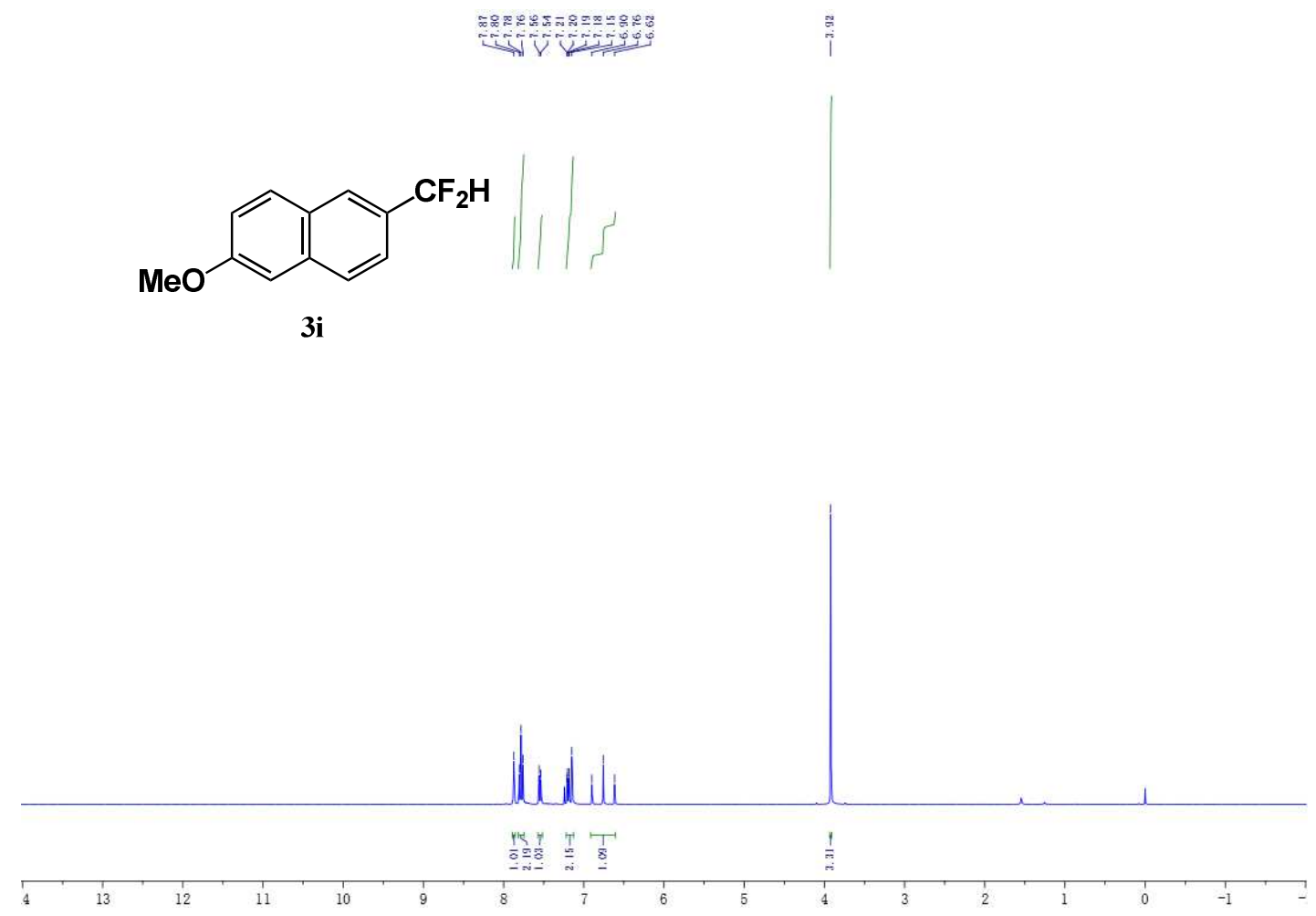

${ }^{19}$ F NMR

$z=$
$\frac{8}{4}$<smiles>COc1ccc2cc(C(F)F)ccc2c1</smiles>

\begin{tabular}{lllllllllllllllllllllllllllllll}
\hline & 1 \\
30 & 20 & 10 & 0 & -10 & -20 & -30 & -40 & -50 & -60 & -70 & -80 & -90 & -100 & -110 & -120 & -130 & -140 & -150 & -160 & -170 & -180 & -190 & -200 \\
\hline
\end{tabular} 


\section{${ }^{13}$ C NMR}<smiles>COc1ccc2cc(C(F)F)ccc2c1</smiles>
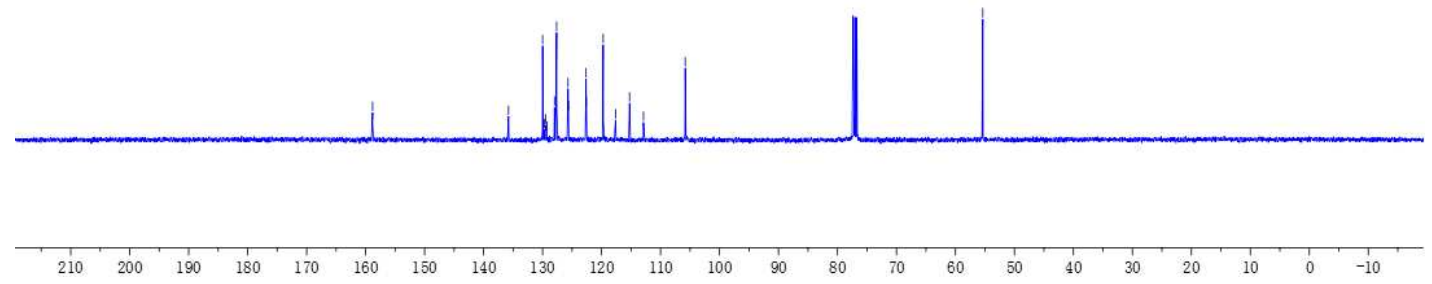

\section{${ }^{1}$ H NMR}
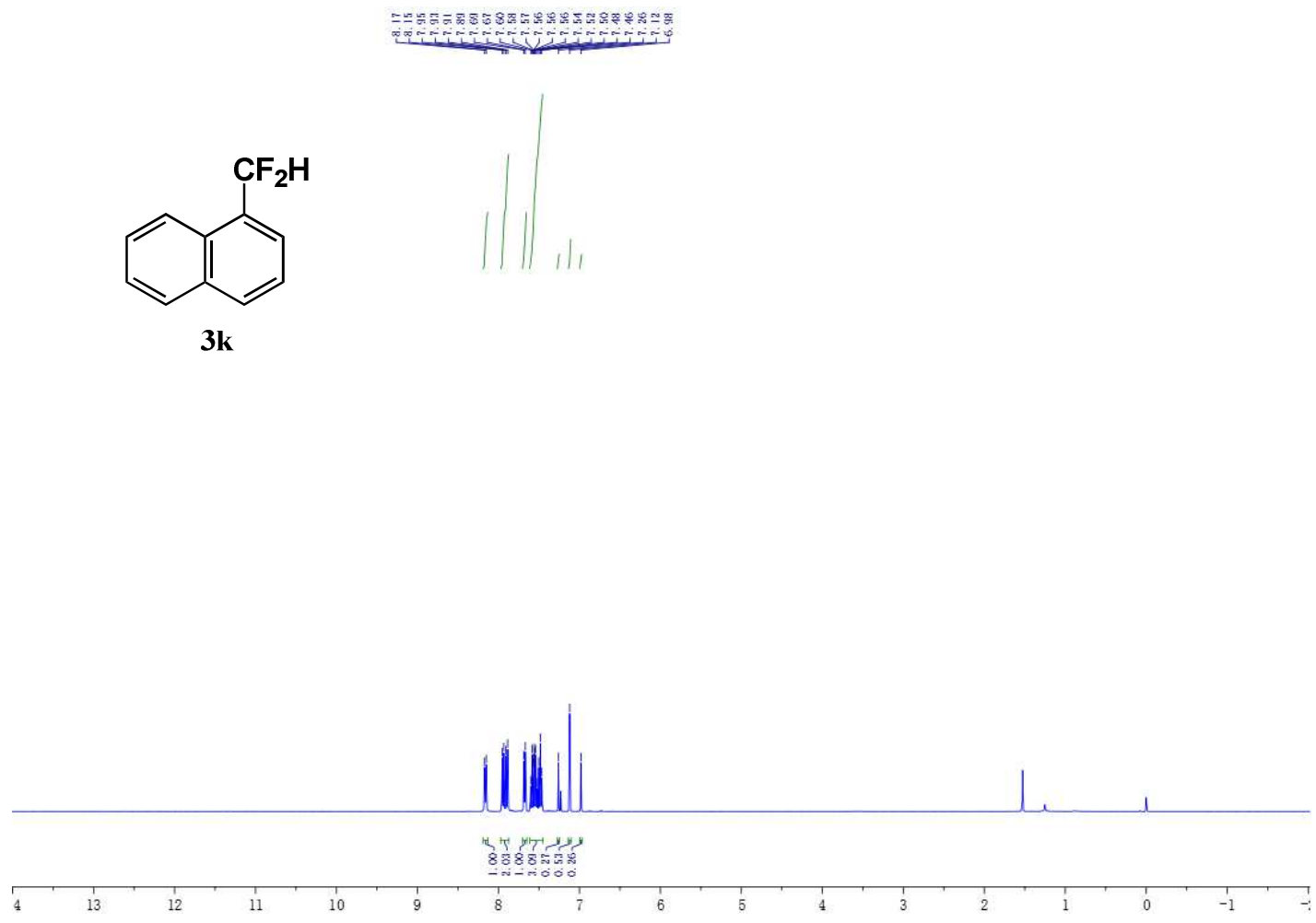


\section{${ }^{19}$ F NMR}

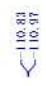<smiles>FC(F)(F)c1cccc2ccccc12</smiles>

$3 k$

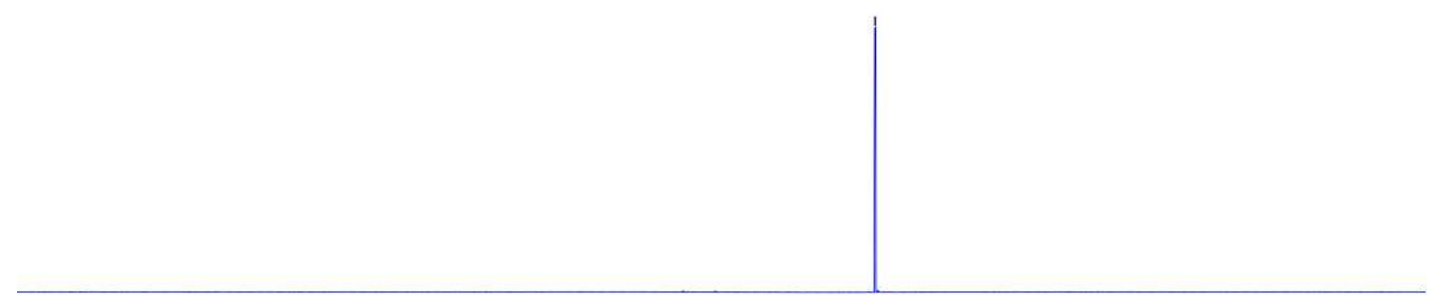

\begin{tabular}{llllllllllllllllllllllllllllll}
\hline & 1 & 10 & 10 & 0 & -10 & -20 & -30 & -40 & -50 & -60 & -70 & -80 & -90 & -100 & -110 & -120 & -130 & -140 & -150 & -160 & -170 & -180 & -190 & -200 \\
\hline
\end{tabular}

\section{${ }^{1}$ H NMR}
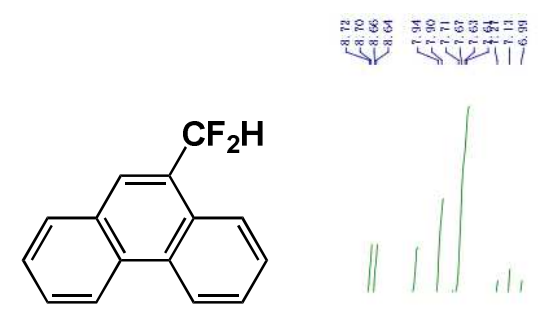

31

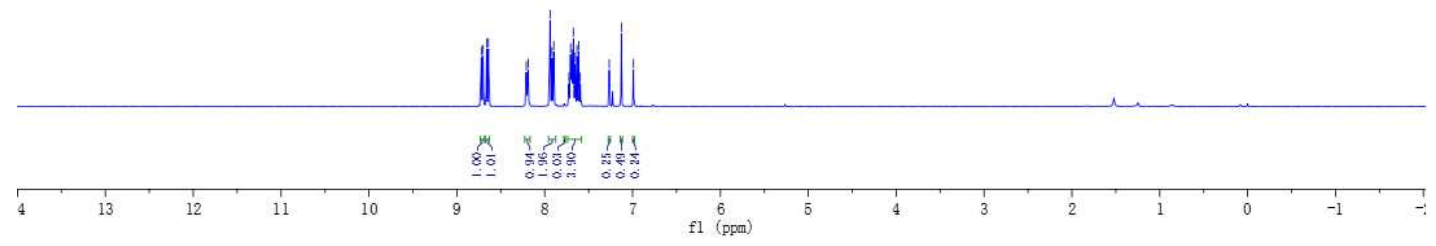




\section{${ }^{19}$ F NMR}
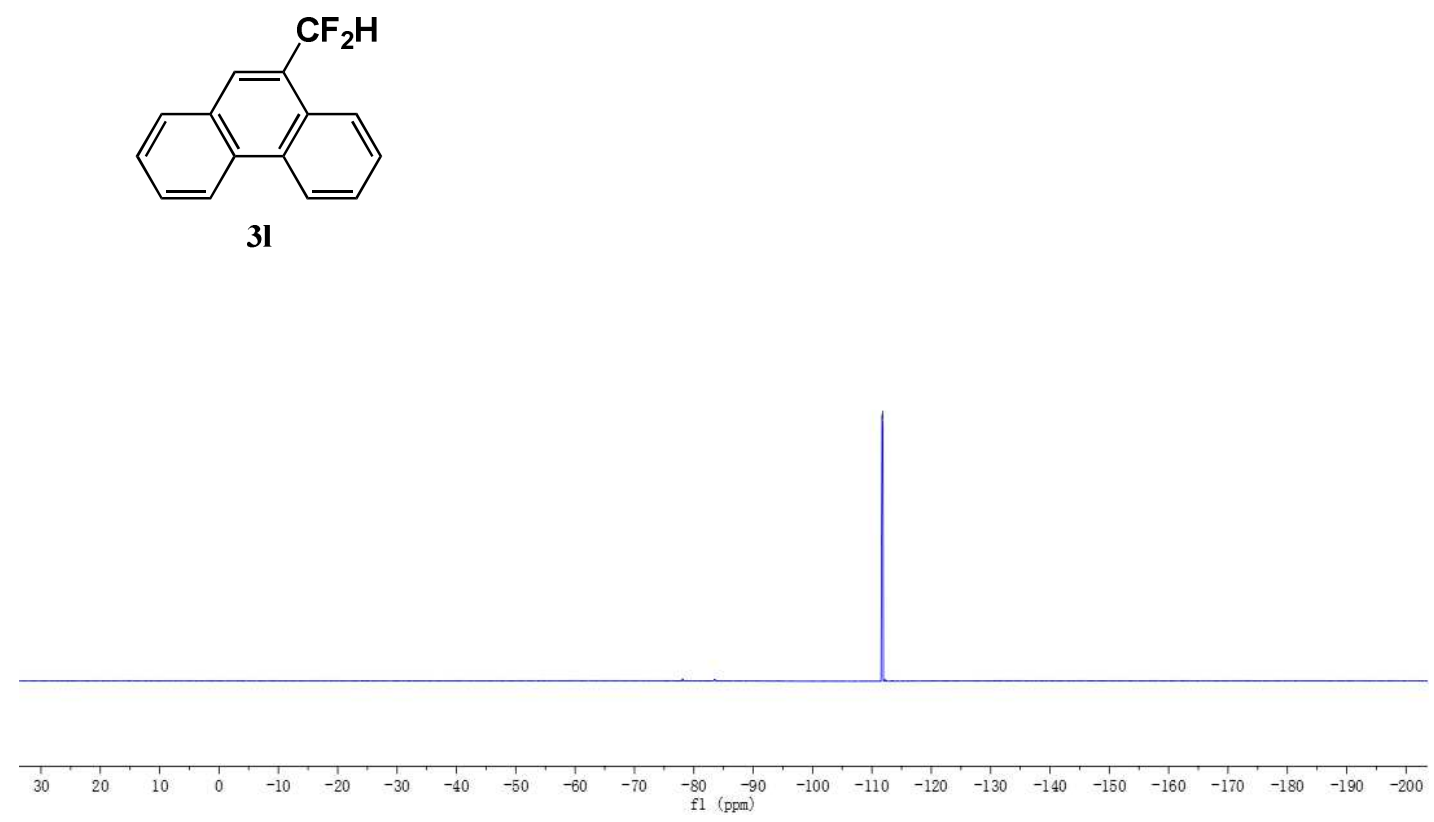

\section{${ }^{1}$ H NMR}
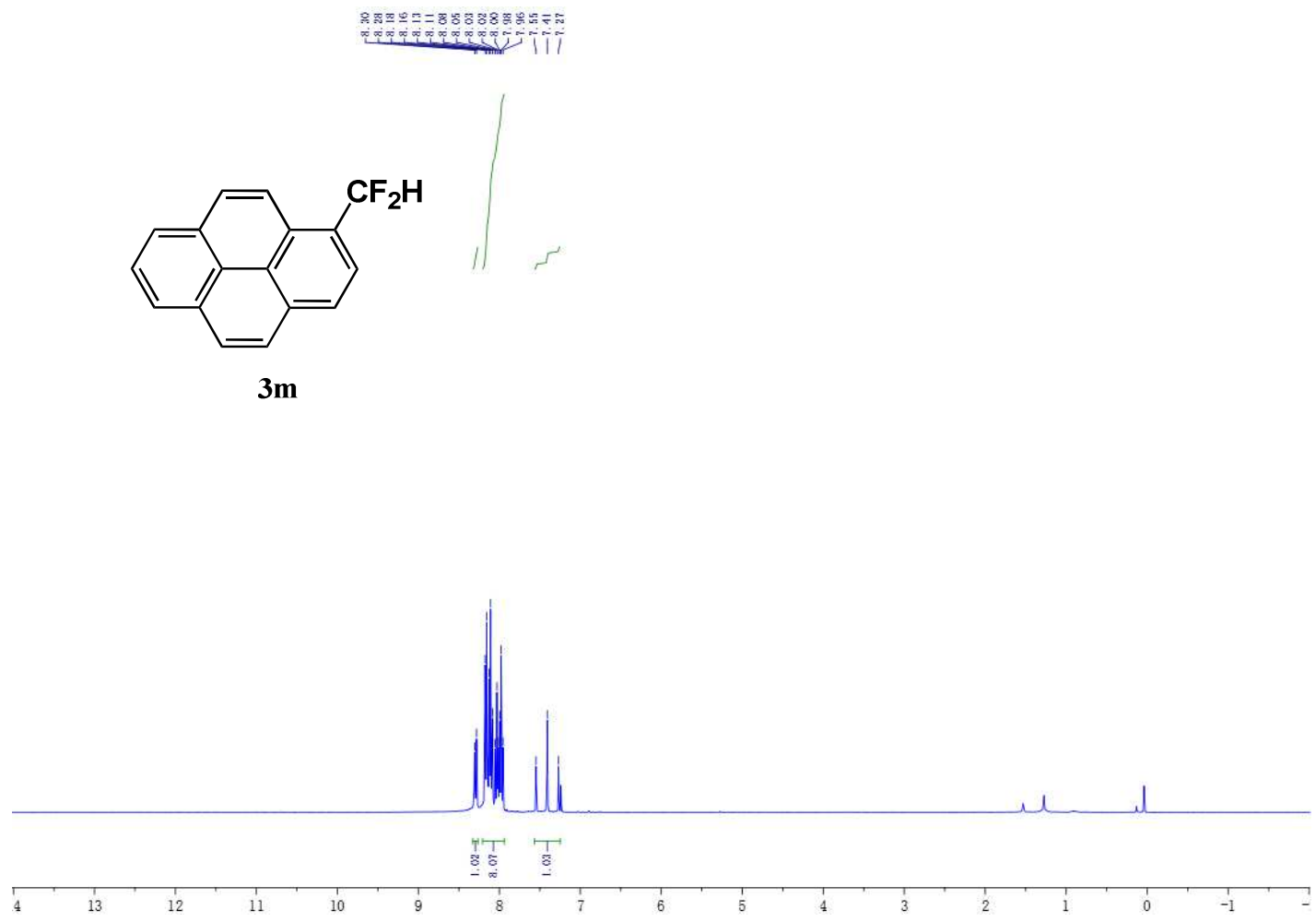


\section{${ }^{19}$ F NMR}

$z=5$
$\frac{8}{8}$
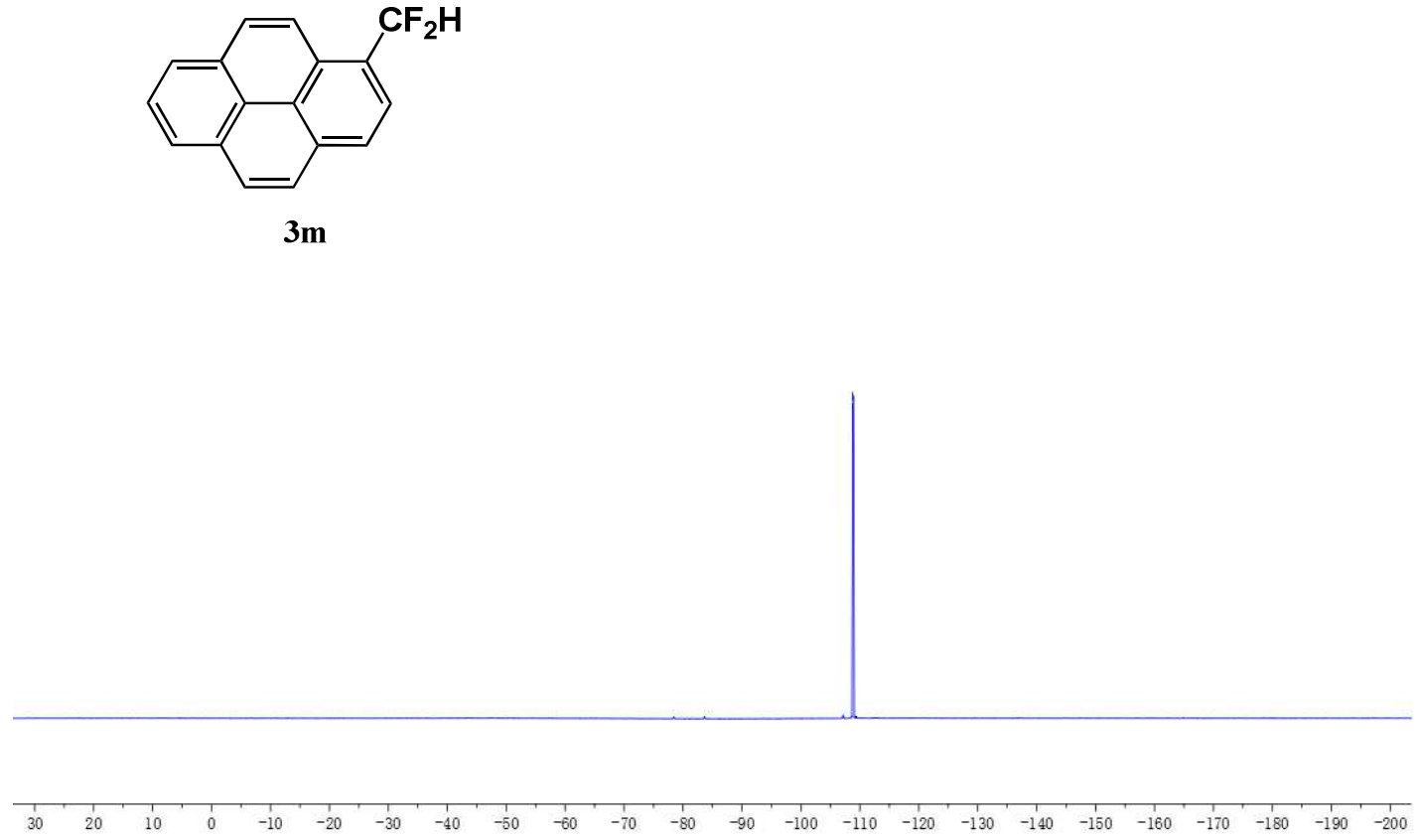

${ }^{13}$ C NMR

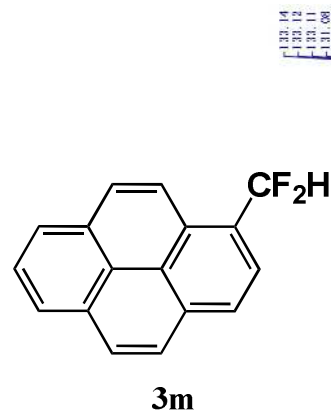




\section{${ }^{1}$ H NMR}
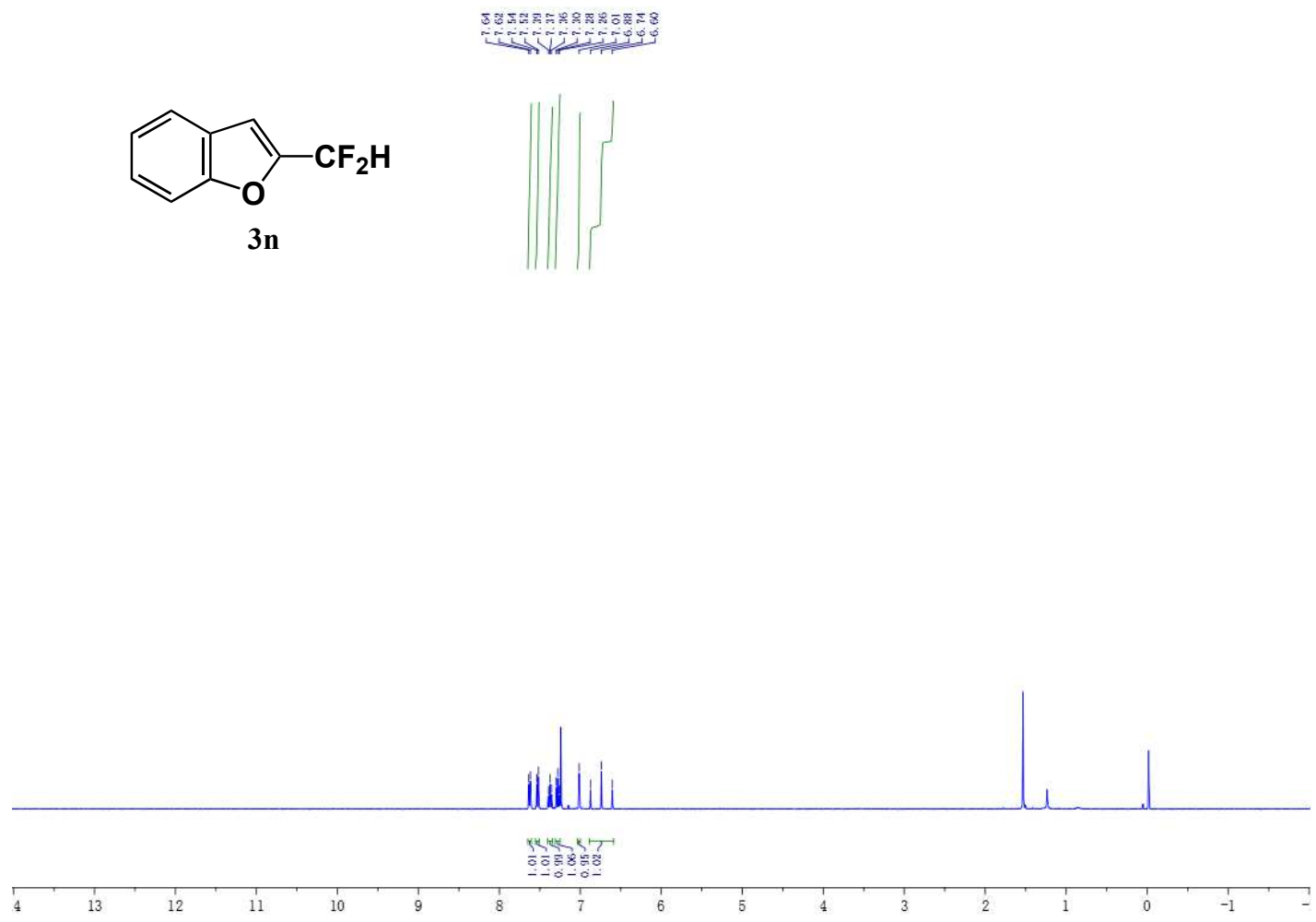

\section{${ }^{19}$ F NMR}
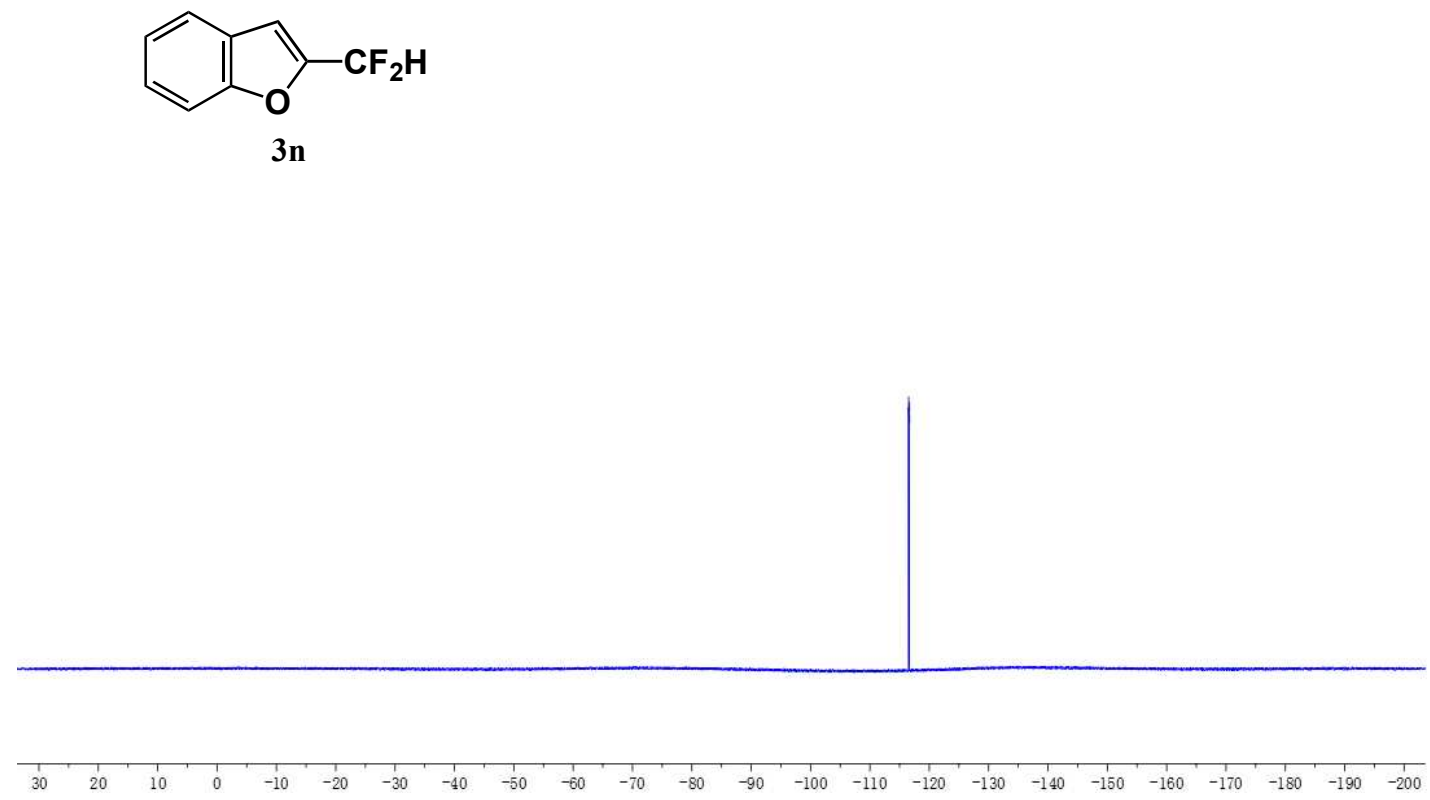


\section{${ }^{1}$ H NMR}
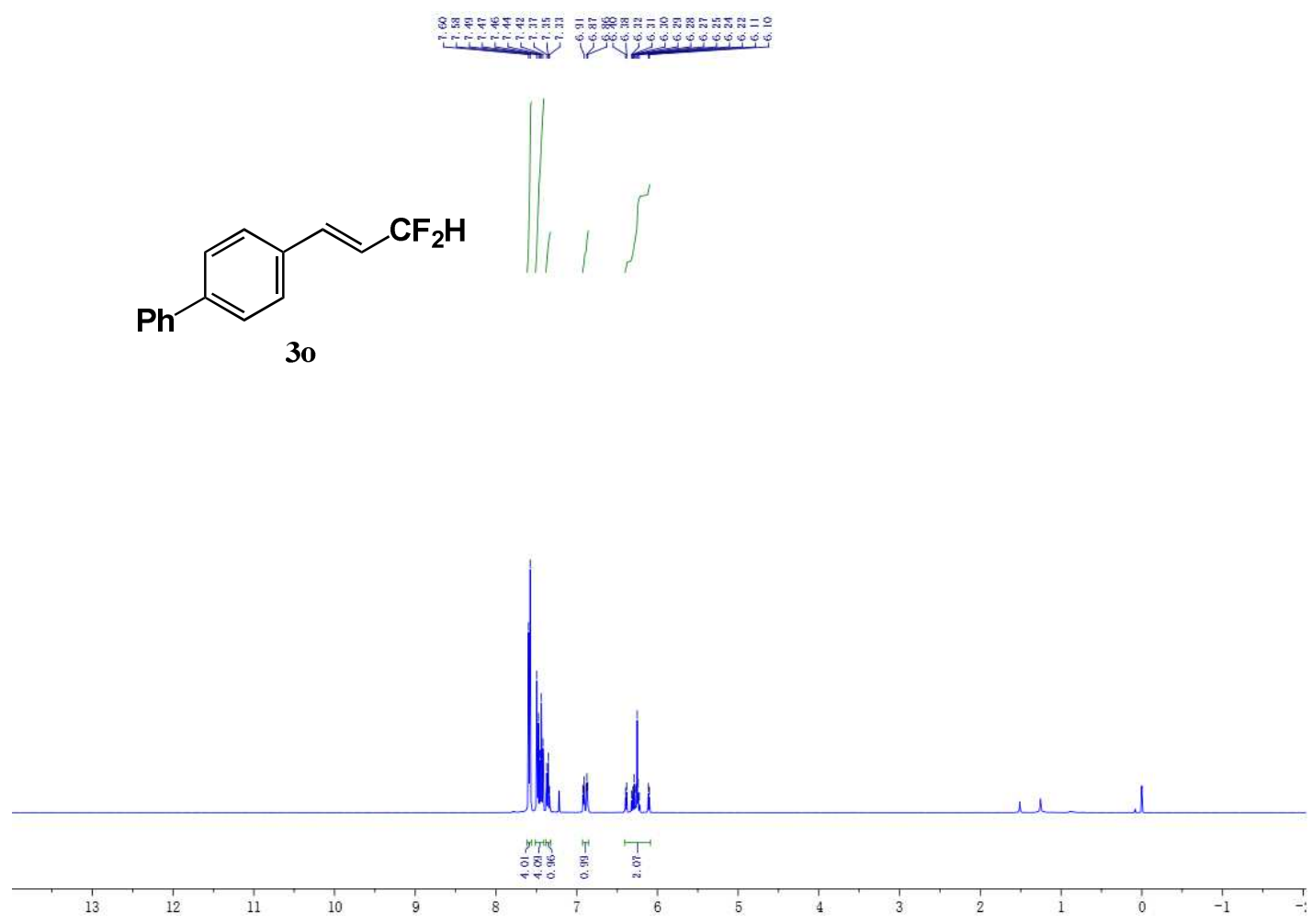

\section{${ }^{19}$ F NMR}

P.F

8 $88 \frac{8}{8}$
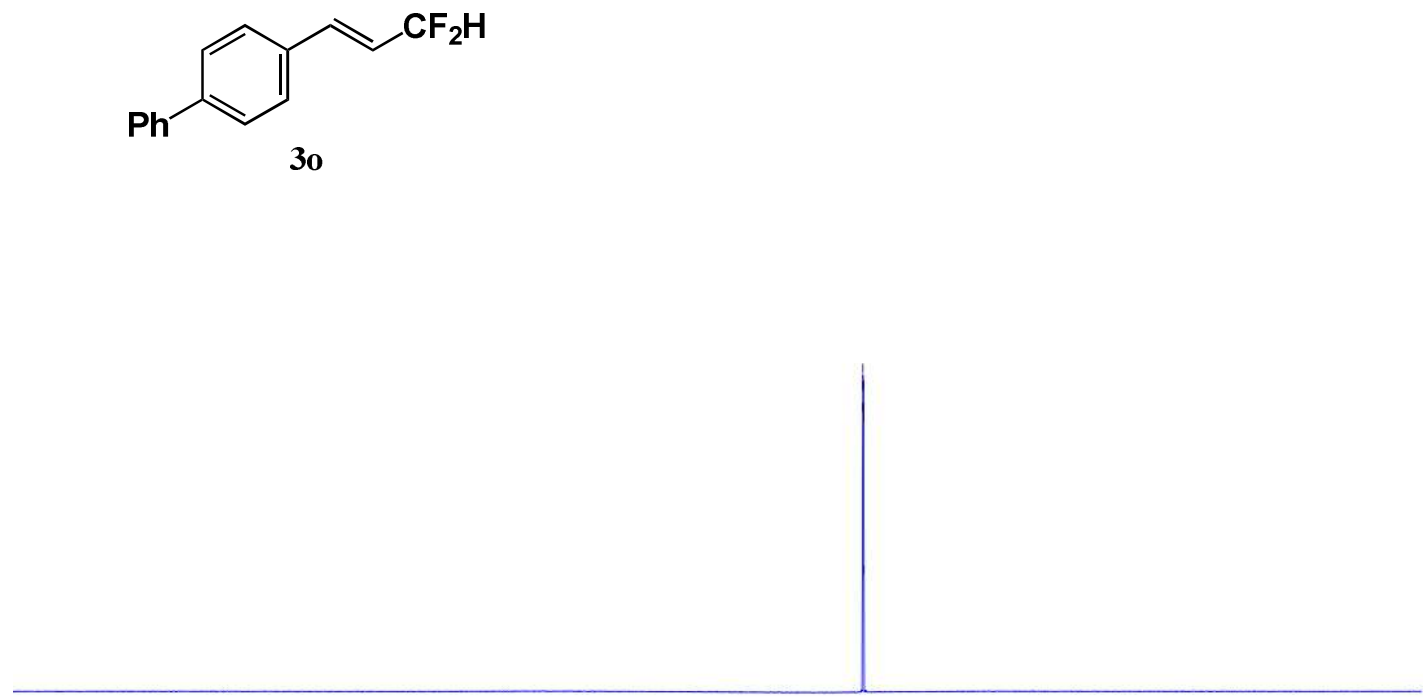

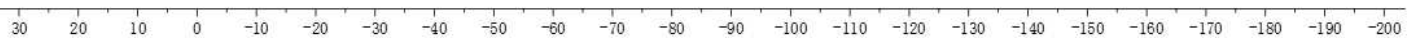




\section{${ }^{13}$ C NMR}

곤

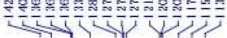

$\overbrace{30}^{C F_{2} H}$

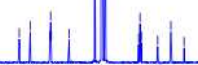

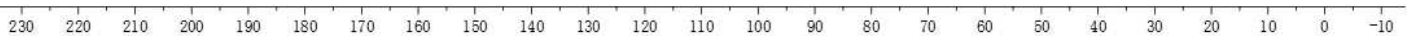

\section{${ }^{1}$ H NMR}

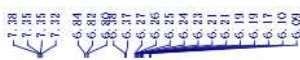
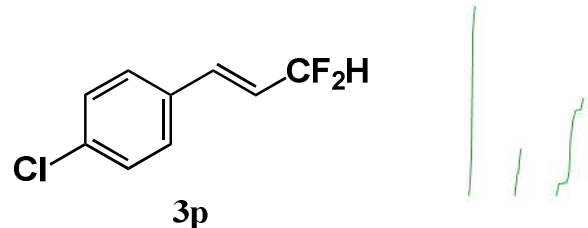

$3 p$

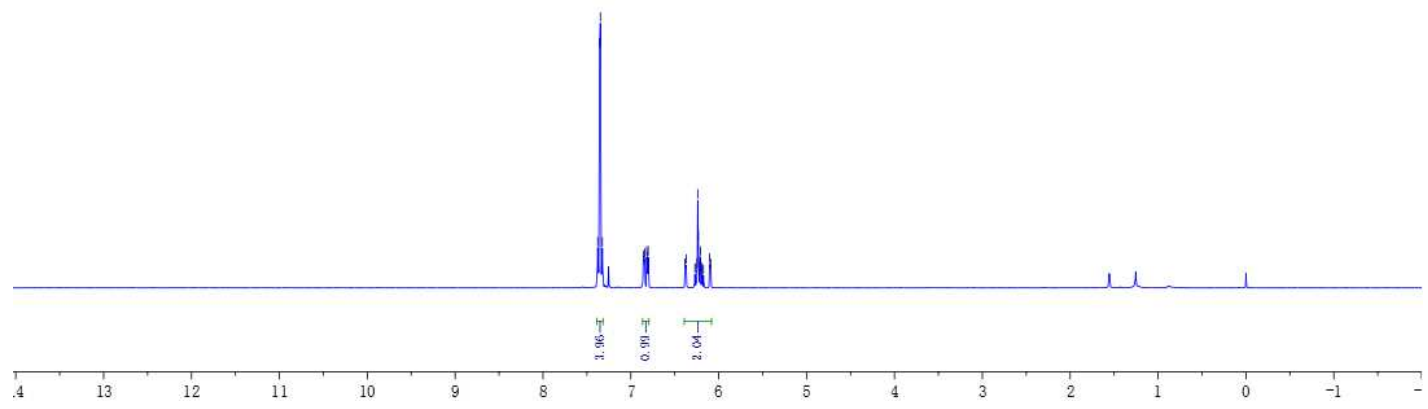




\section{${ }^{19}$ F NMR}

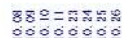

起<smiles>FC(F)(F)C=Cc1ccc(Cl)cc1</smiles>

\begin{tabular}{lllllllllllllllllllllllllll}
\hline & 1 & 20 & 10 & 0 & -10 & -20 & -30 & -40 & -50 & -60 & -70 & -80 & -90 & -100 & -110 & -120 & -130 & -140 & -150 & -160 & -170 & -180 & -190 & -200 \\
\hline
\end{tabular}

\section{${ }^{1}$ H NMR}

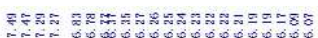

Viv iั

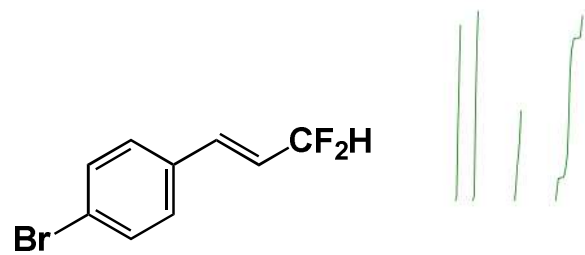

$3 q$

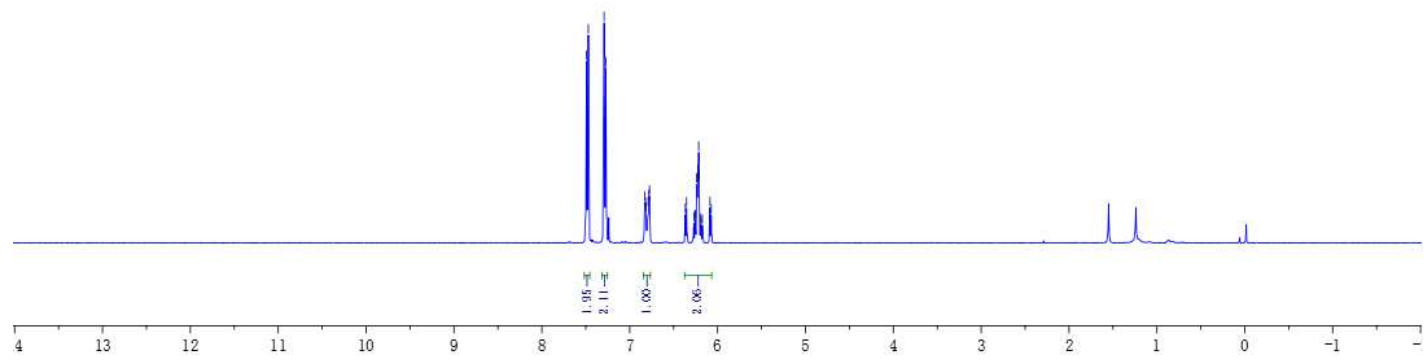




\section{${ }^{19}$ F NMR}

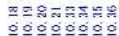

近<smiles>FC(F)(F)C=Cc1ccc(Br)cc1</smiles>
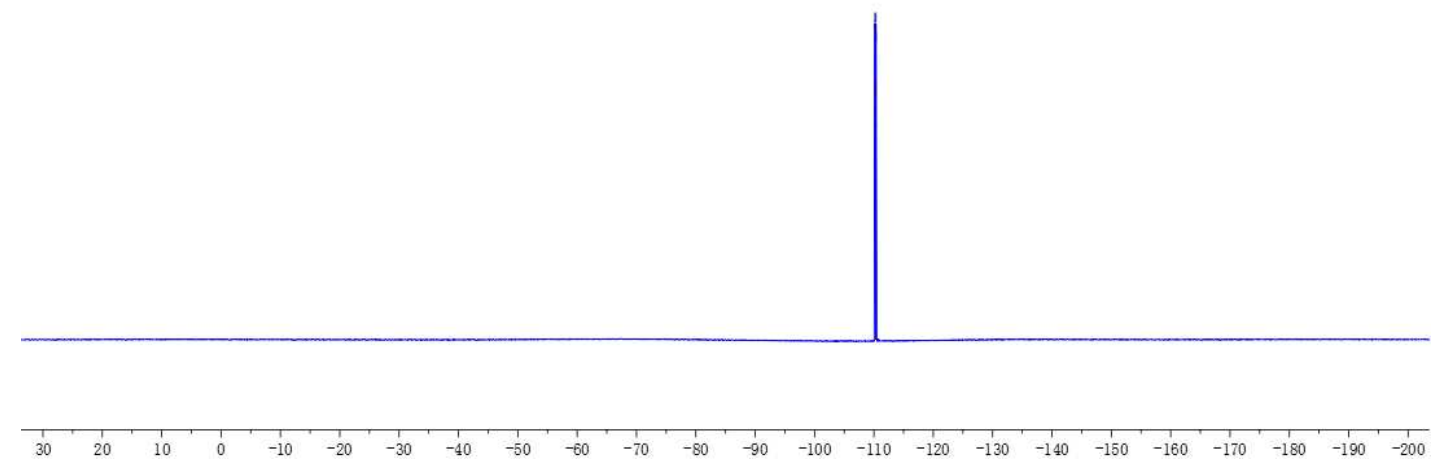

\section{${ }^{1}$ H NMR}

\section{5}
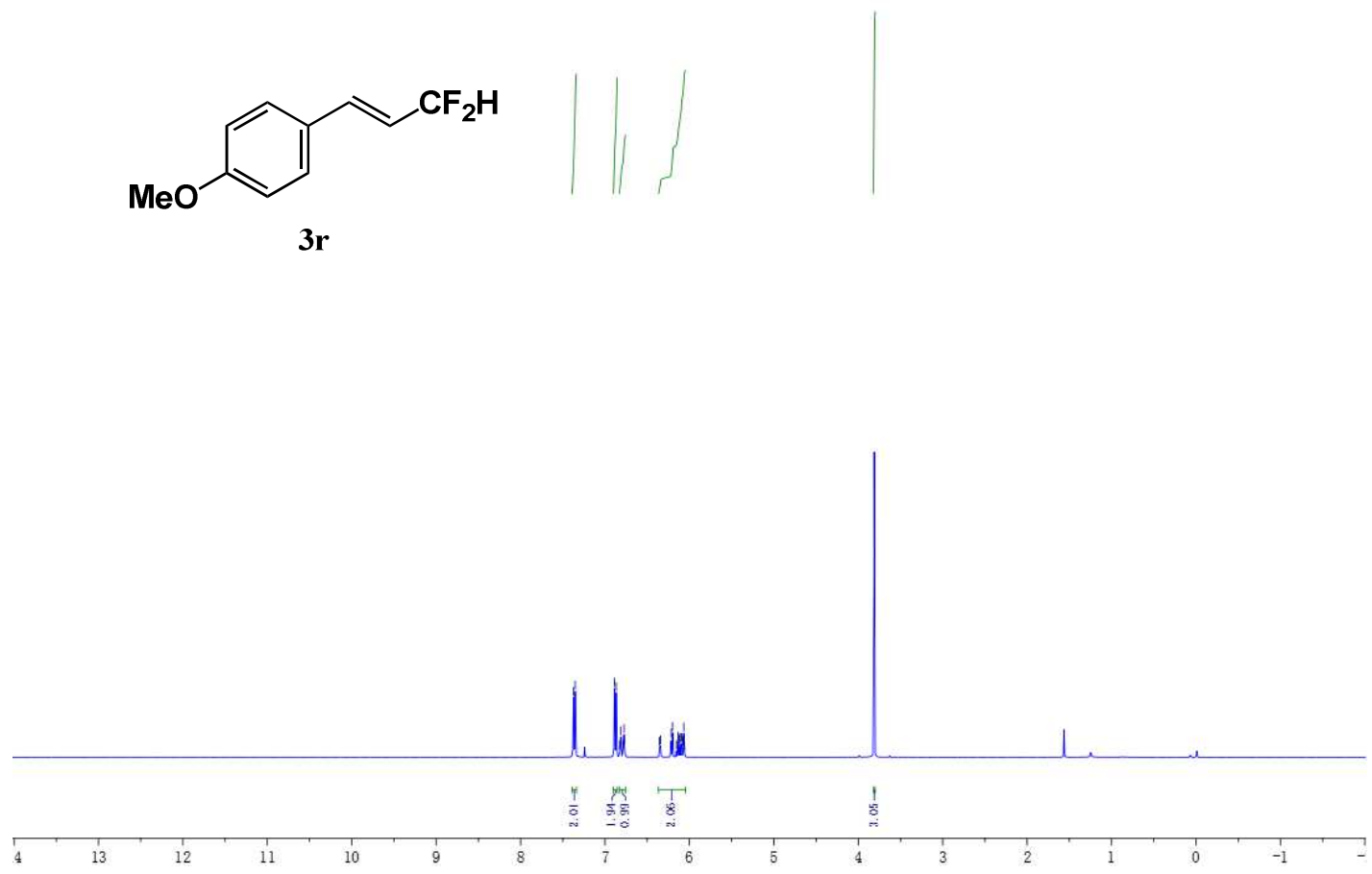


\section{${ }^{19}$ F NMR}

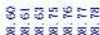

这这
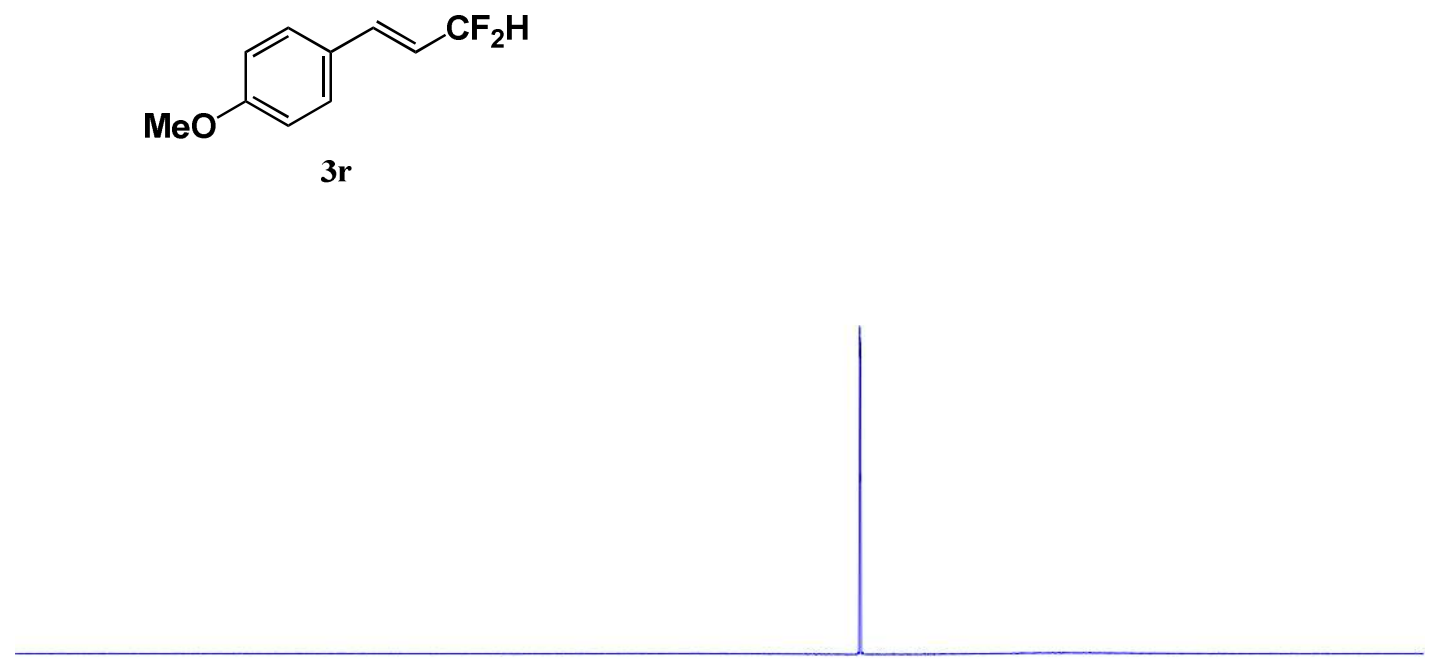

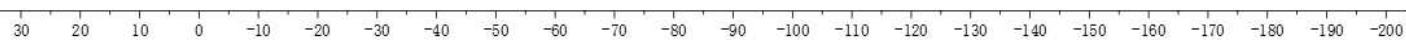

\section{${ }^{1}$ H NMR}

Fom

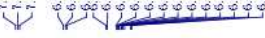

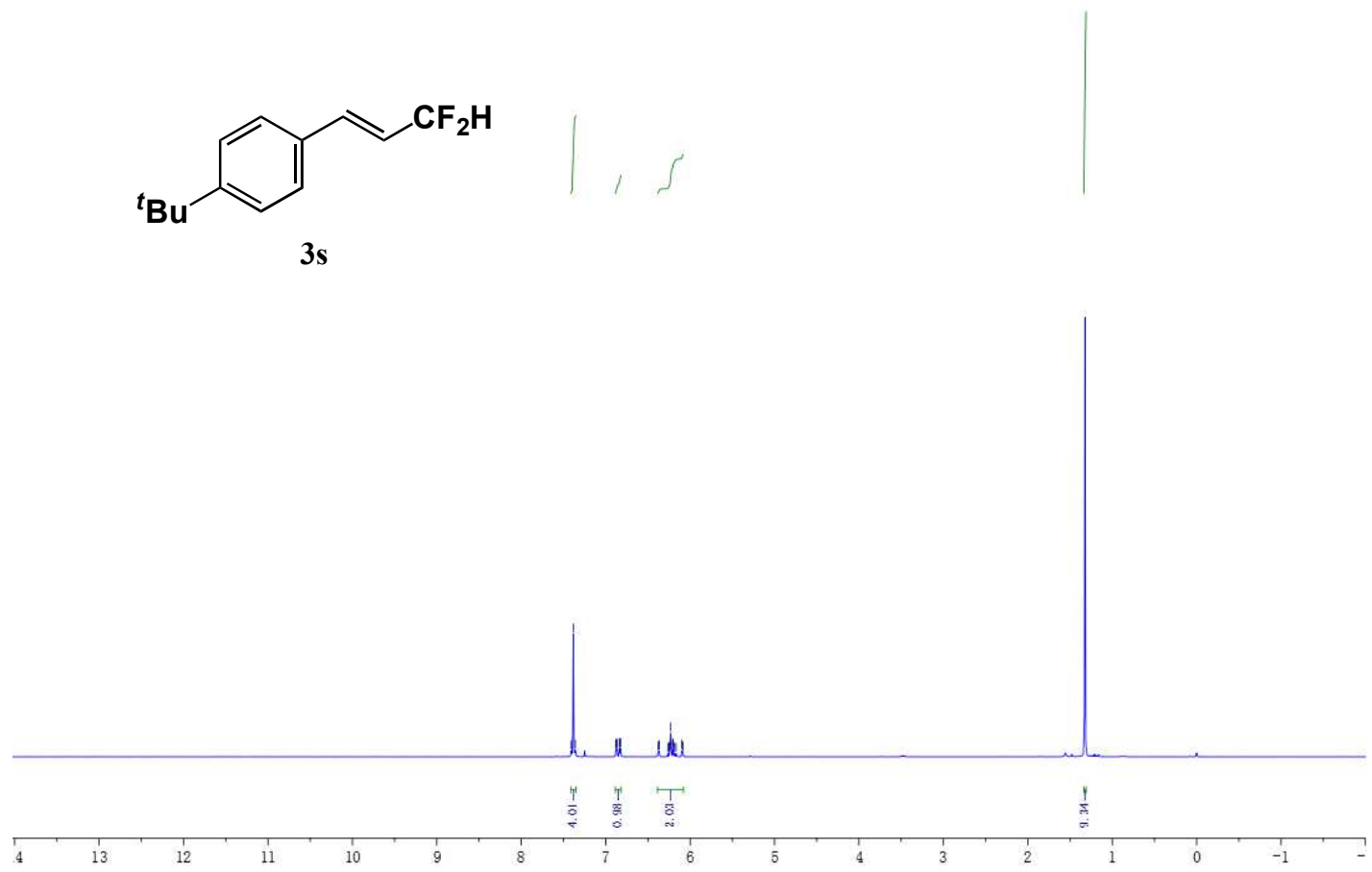




\section{${ }^{19}$ F NMR}

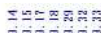

$48 \frac{8}{2}$

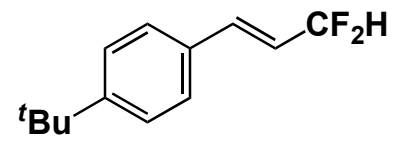

$3 s$

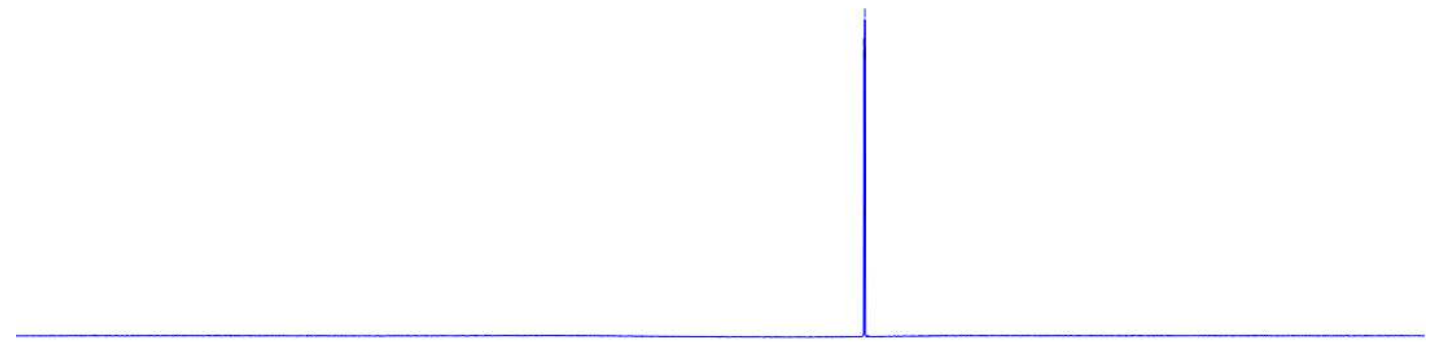

$\begin{array}{llllllllllllllllllllllllllll}1 & 1 & 10 & 10 & 0 & -10 & -20 & -30 & -40 & -50 & -60 & -70 & -80 & -90 & -100 & -110 & -120 & -130 & -140 & -150 & -160 & -170 & -180 & -190 & -200\end{array}$

\section{${ }^{1}$ H NMR}
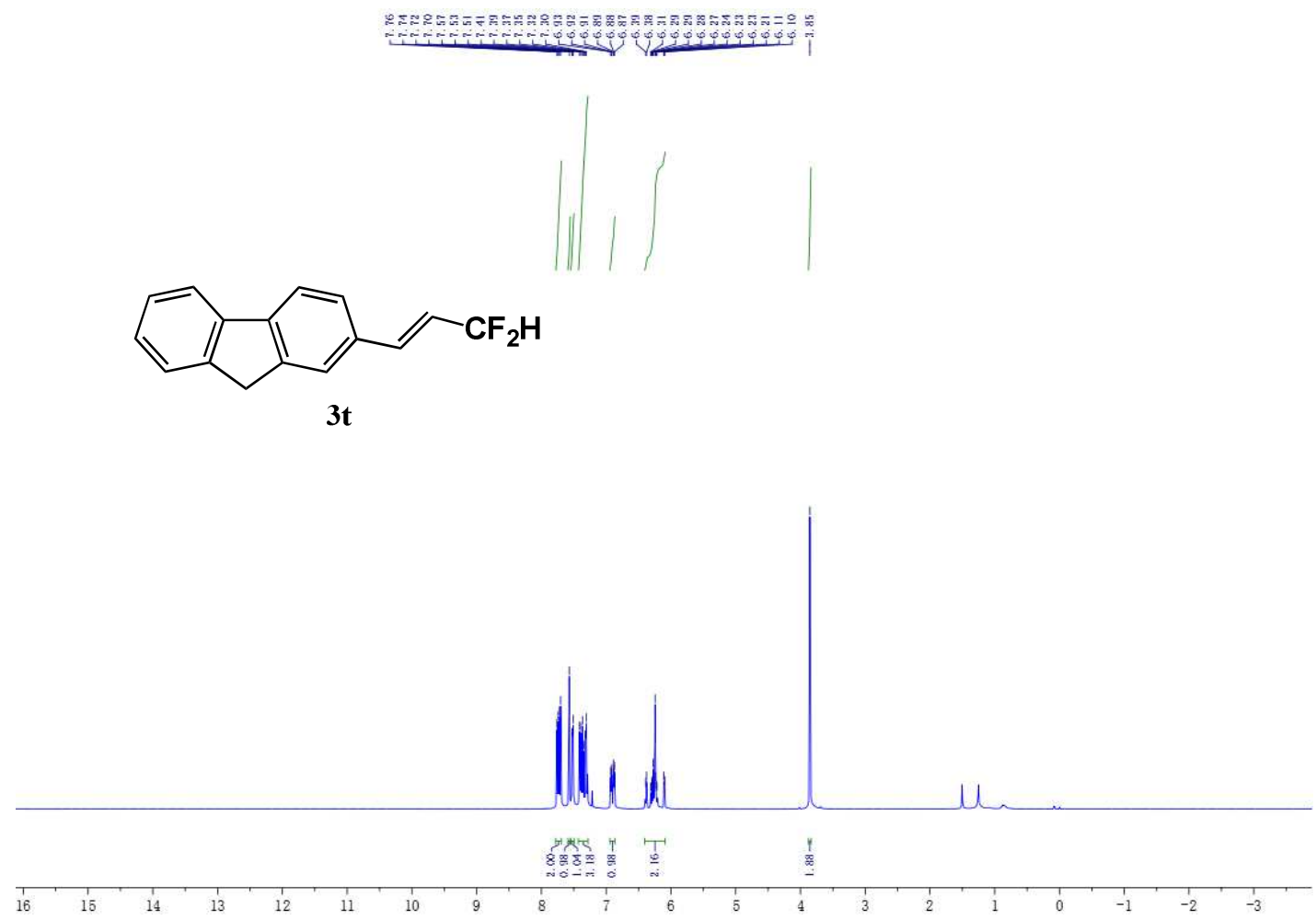


\section{${ }^{19}$ F NMR}
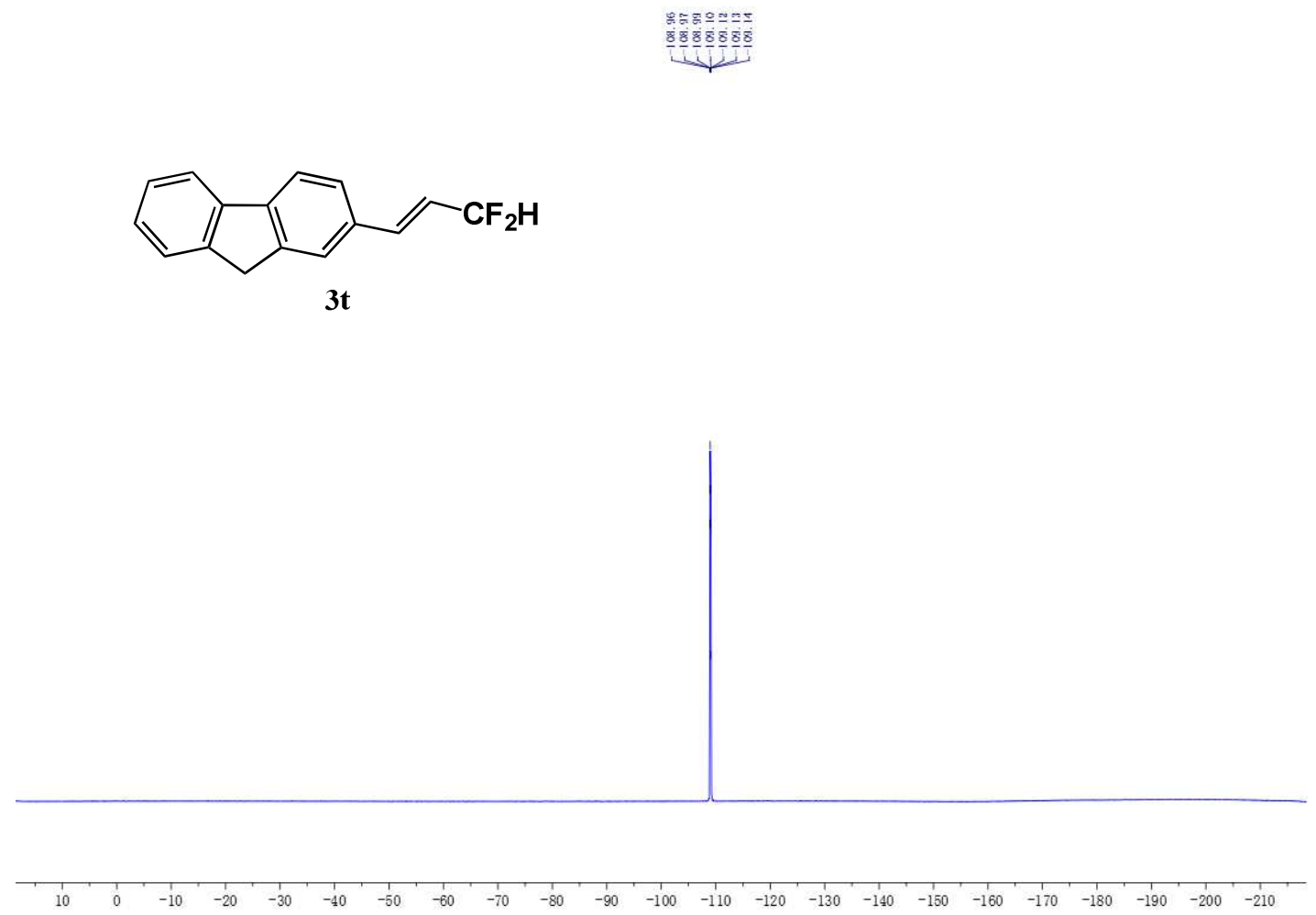

${ }^{13}$ C NMR
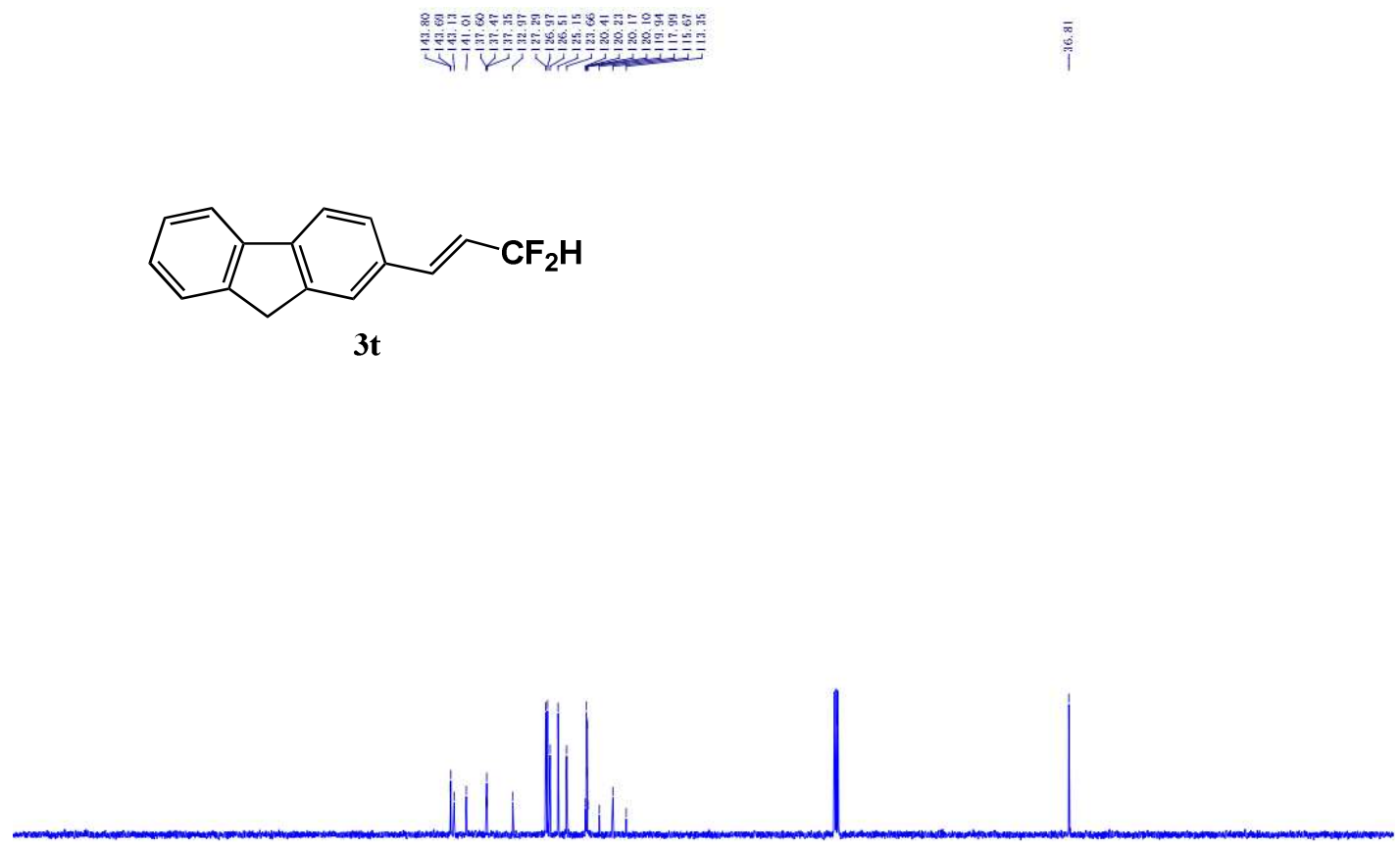

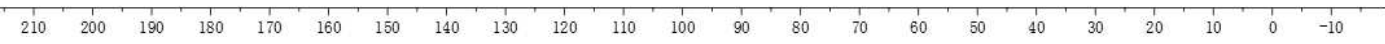




\section{${ }^{1}$ H NMR}

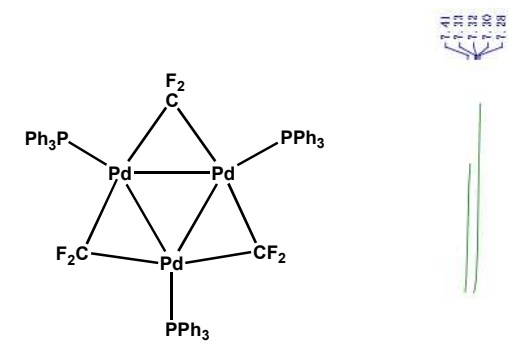

A

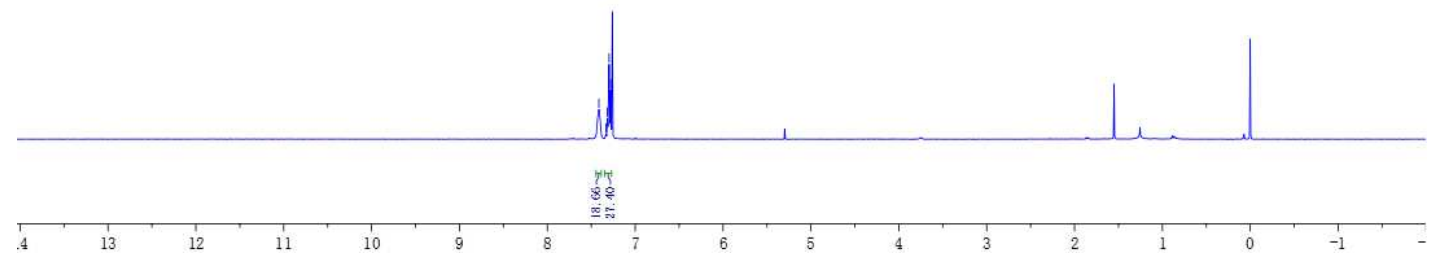

${ }^{19}$ F NMR
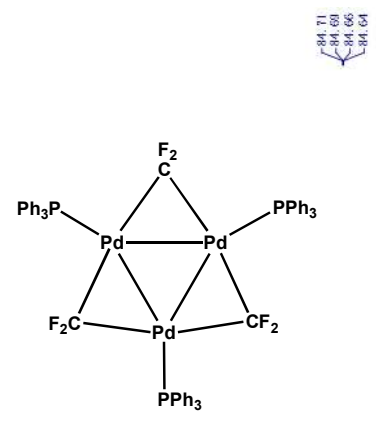

A

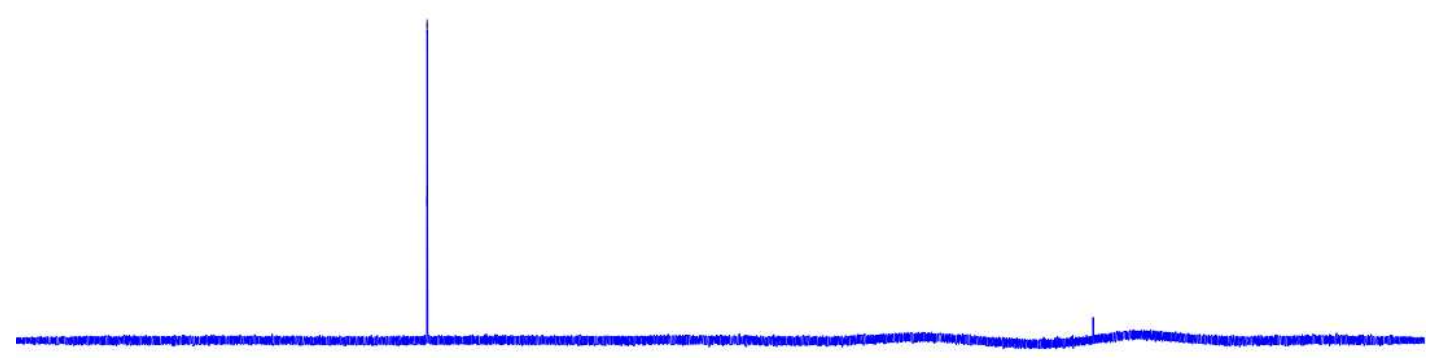

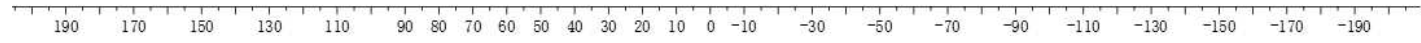


${ }^{31}$ P NMR

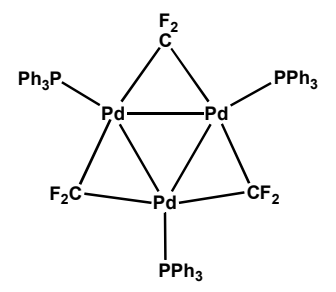

A

1.

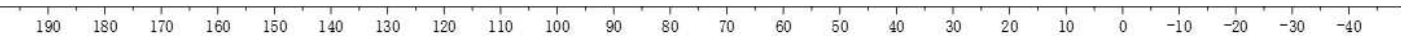

